\title{
Inference Rules in Nelson's Logics, Admissibility and Weak Admissibility
}

\author{
Sergei Odintsov and Vladimir Rybakov
}

\begin{abstract}
Our paper aims to investigate inference rules for Nelson's logics and to discuss possible ways to determine admissibility of inference rules in such logics. We will use the technique offered originally for intuitionistic logic and paraconsistent minimal Johannson's logic. However, the adaptation is not an easy and evident task since Nelson's logics do not enjoy replacement of equivalences rule. Therefore we consider and compare standard admissibility and weak admissibility. Our paper founds algorithms for recognizing weak admissibility and admissibility itself - for restricted cases, to show the problems arising in the course of study.
\end{abstract}

Mathematics Subject Classification (2010). Primary 03F99; Secondary $03 \mathrm{~B} 53$.

Keywords. Nelson's logic, strong negation, admissible inference rules, weak admissibility, decidability, Vorob'ev translation.

\section{Introduction}

In the area of non-classical logics, besides discussions concerning efficient axiomatizations, the question of applicability possible new inference rules, different from standard ones, was set up in 1950x by P.Lorenzen. In particular, he, it seems, first time formulated the concept of admissibility for inference rules in precise terms. The admissibility problem (to determine for any given rule if this rule is admissible for a given logic) was in focus of interest for many logicians. Active research in the area may be dated to Harvey Friedman problem [15]: if there is an algorithm for verification of admissibility in the intuitionistic propositional logic IPC (this problem was first solved by Rybakov in 1984, [29]). Then the admissibility has been investigated from various viewpoints for many different logical systems related

This work was supported by Russian Foundation for Basic Research, project No. 12-0100168-a. 
to non-classical propositional logics (cf. V. Rybakov [30, 31, 32, 33, 35, 34], Rybakov, et al [2, 3], R. Iemhoff [4, 5], R. Iemhoff and G. Metcalfe [6], E. Jerabek $[8,11,9,7])$. Algorithmic problem of recognizing admissibility, problem of descriptions bases for admissible rules, and many other related questions were considered. For example, only a necessary condition for admissibility of inference rules in the branching-time temporal logic $T_{S 4}$ was found in [36], though for linear temporal logic LTL the problem was solved in full [34]. Complexity problem for admissibility in intuitionistic logic and some modal logics was first studied and re-solved in Jerabek [10]. A new approach to study admissible rules was offered by S. Ghilardi via unification technique. He in $([12], 1999)$ first found an algorithm writing out a complete set of unifiers for any unifiable in IPC formula, and this gives another solution for admissibility problem.

This our article continues the investigation of admissible rules in paraconsistent logics [25], which was started in [24]. In that paper the problematic of admissibility and unification was addressed to minimal Johansson's logic $\mathbf{J}[16]$ and positive intuitionistic logic $\mathbf{I P C} \mathbf{C}^{+}$. The first of these logics has the negation weaker then the intuitionistic one, whereas the second logic simply lacks the negation connective. The most essential difficulties arising in the study of admissibility for such logics is connected with the impossibility to use the Gödel-Tarski translation. In case of intuitionistic logic, the problem of admissibility for inference rules with parameters was solved earlier by V. Rybakov in [31], where the proof was essentially based on the GödelTarski translation of intuitionistic logic into $S 4$. In [24], we presented the construction which does not appeal to modal language, and due to this reason modifications of this construction can be applied to a wide class of logics based on the positive fragment of intuitionistic logic. In [24], we adopted this technique to solve the admissibility and unification problems for positive intuitionistic logic $\mathbf{I} \mathbf{P C}^{+}$. In the present article we make the first effort to study the admissibility problem for extensions of Nelson's logic and give further applications of the construction developed in [24].

To overcome the non-constructivity of intuitionistic negation D. Nelson [19] suggested the concept of constructible falsity, which assumes that the falsity of atomic statements is given explicitly, and the falsity of complex statements is reduced to the truth or falsity of its constituents via a constructive procedure. Subsequently, his system of constructive logic with strong negation, traditionally denoted by N3, was axiomatized by Vorob'ev $[38,39]$ and studied algebraically by Helena Rasiowa [26, 27].

The concept of constructible falsity agrees well with that of paraconsistency. If the falsity of an atom $p$ represented as $\sim p$, the strong negation of $p$, is given explicitly, we may admit that both $p$ and $\sim p$ are true. The paraconsistent Nelson's logic N4 is obtained by deleting the "explosive" axiom $\sim p \rightarrow(p \rightarrow q)$ from the axiomatics of N3. From the early 1970s several versions of N4 were studied independently by R. Routley (later R. Sylvan) in the propositional case in [28], by López-Escobar in [18] and by Nelson himself 
in [1], both in the first-order case. Algebraic semantics for $\mathbf{N} 4$ was suggested in $[20]$.

Kripke semantics for N3 ( $[37,14])$ is readily obtained from the usual Kripke semantics for intuitionistic logic by assigning to each world, instead of a set of atoms, a set of literals, i.e. atoms or strongly negated atoms. Equivalently, the truth-assignment on atoms and worlds is 3 -valued, to reflect the three cases of verified, falsified or neither. Changing to 4-valued assignments in Kripke models produces a semantics for $\mathbf{N 4}$ : the fourth value now corresponds to "overdetermined" or the situation that both a literal and its contrary are verified at a world. This explains the choice of denotation N3 and N4. An alternative possible worlds semantics for constructive logic with strong negation was provided by Routley in [28]. The strong negation is interpreted in Routley frames via *-operator, which is typical for relevant logics. In this paper, we will consider Kripke frames augmented with the valuation of literals. This kind of semantics is obviously equivalent to the semantics with four-valued assignment and fits well to the constructions from [24].

We will consider the paraconsistent Nelson's logic N4 and several extensions of the logic $\mathbf{N} \mathbf{4}^{\perp}$ [21]. This is a version of paraconsistent Nelson's logic obtained from $\mathbf{N} 4$ by adding to the language the intuitionistic falsity constant $\perp$ allowing to define the intuitionistic negation as $\neg \varphi:=\varphi \rightarrow \perp$. The combination of paraconsistent negation $\sim$ and explosive negation $\neg$ in the logic $\mathbf{N} \mathbf{4}^{\perp}$ is similar to the situation in logic programming, where logic programs with two kinds of negation are considered: the traditional for logic programming default negation (corresponding to the intuitionistic negation) and the explicit negation based on the idea similar to that of constructible falsity. In [23], it was proved that the logic $\mathbf{N}_{\mathbf{9}}$, a nine-valued $\mathbf{N} \mathbf{4}^{\perp}$-extension, serve as a deductive base for paraconsistent answer set semantics of logic programs with two kinds of negation. On the other hand, adding the constant $\perp$ to the language of $\mathbf{N} 4$ results in a more regular structure of the class of $\mathbf{N} 4^{\perp}$-extensions. As it was shown in [21], to clarify the general structure of the class $\mathcal{E} \mathbf{N} 4^{\perp}$ of $\mathbf{N} 4^{\perp}$-extensions it is essential to distinguishe in this class the subclasses Exp of explosive logics, Nor of normal logics, and Gen of logic in general form. In this paper, we consider the admissibility problem for the logics $\mathbf{N} 3, \mathbf{N} 4^{\mathrm{N}}, \mathbf{N} 4^{\circ}$, and $\mathbf{N} 4^{\perp}$, which are the least logics of classes Exp, Nor, Exp $\cap$ Nor, and respectively Gen.

We cannot yet solve the general admissibility problem for Nelson's logics, but we can explicitly describe the strengthening of admissibility problem for positive and intuitionistic logics which is equivalent to the admissibility problem for Nelson's logics $\mathbf{N} 4$ and $\mathbf{N} 4^{\perp}$ respectively. On this way we come to the notion of dependent admissibility, which assumes the connections between variables in the premiss and conclusion of the rule. The connected variables must be replaced by formulas the structures of which are agreed in one or another way. In the version of dependent admissibility equivalent to the admissibility problem in Nelson's logics we have to divide all variables 
into ordered pairs, and replaced each pair of connected variables by a formula and its dual.

The main feature of Kripke semantics of Nelson's logic is the independence of interpretation of literals $p$ and $\sim p$. Interpretations $p$ and $\sim p$ are completely independent in case of the logics $\mathbf{N} 4$ and $\mathbf{N} 4^{\perp}$, if we pass to extensions of these logics, some restriction on this pair of interpretations must be imposed. For example, $V(p)$ and $V(\sim p)$ have empty intersection in case of $\mathbf{N 3}$, or $V(p) \cup V(\sim p)$ is dense in the algebra of cones of a Kripke frame in case of $\mathbf{N} \mathbf{4}^{\perp}$. These observations lead to the notion of weak admissibility, according to which we replace positive and negative occurrences of a variable via different formulas. This pair of formulas is arbitrary in case of $\mathbf{N 4}$ and $\mathbf{N} 4^{\perp}$ and satisfies some restrictions for proper $\mathbf{N} \mathbf{4}^{\perp}$-extensions. For example, elements of such pair must be consistent in case of N3. We formulate the weak admissibility problem for all mentioned logic and show that for the logics $\mathbf{N} 4$ and $\mathbf{N} 4^{\perp}$ this problem is equivalent to the admissibility problem for logic $\mathbf{I n t}^{+}$and Int respectively. In this way the fact that the weak admissibility problem for $\mathbf{N} 4$ and $\mathbf{N} 4^{\perp}$ is decidable follows from the results of [24]. In case of logics $\mathbf{N 3}, \mathbf{N} 4^{\mathrm{N}}$, and $\mathbf{N} 4^{\circ}$ there is no simple reduction to earlier obtained results, and we have to adapt the construction from [24] to prove that the weak admissibility problem for these logics is decidable.

\section{Nelson's logics}

Fix a countable set of propositional variables $\operatorname{Prop}=\left\{p_{1}, p_{2}, \ldots, p_{n}, \ldots\right\}$ and an additional set of propositional variables $\operatorname{Prop}^{\dagger}=\left\{p_{1}^{\dagger}, p_{2}^{\dagger}, \ldots, p_{n}^{\dagger}, \ldots\right\}$. By a propositional language we mean a finite set of connectives with indicated arities. The set of formulas of a propositional language $\mathcal{L}$ is obtained in a usual way from variables of Prop with the help of connectives of $\mathcal{L}$. In some specially designated cases we will consider also an extended set of formulas defined over the extended set of variables Prop $\cup$ Prop ${ }^{\dagger}$.

By a logic in a propositional language $\mathcal{L}$ with $\rightarrow, \in \mathcal{L}$ we mean a set of formulas closed under the rules of substitution and modus ponens. In this case we can define positive (intuitionistic) logic $\mathbf{I P C}^{+}$as the least logic in the language $\mathcal{L}^{+}=\langle\vee, \wedge, \rightarrow\rangle$ containing the following axioms:

1. $p \rightarrow(q \rightarrow p)$

2. $(p \rightarrow(q \rightarrow r)) \rightarrow((p \rightarrow q) \rightarrow(p \rightarrow r))$

3. $(p \wedge q) \rightarrow p$

4. $(p \wedge q) \rightarrow q$

5. $(p \rightarrow q) \rightarrow((p \rightarrow r) \rightarrow(p \rightarrow(q \wedge r)))$

6. $p \rightarrow(p \vee q)$

7. $q \rightarrow(p \vee q)$

8. $(p \rightarrow r) \rightarrow((q \rightarrow r) \rightarrow((p \vee q) \rightarrow r))$

Intuitionistic logic IPC is the least logic in the language

$\mathcal{L}^{\perp}=\langle\vee, \wedge, \rightarrow, \perp\rangle$ containing axioms of positive logic and the axiom $\perp \rightarrow p$. 
In what follows we use the abbreviation $\varphi \leftrightarrow \psi:=(\varphi \rightarrow \psi) \wedge(\psi \rightarrow \varphi)$.

Paraconsistent Nelson's logic $\mathbf{N} 4$ is the least logic in the language $\mathcal{L}^{\sim}=$ $\langle\vee, \wedge, \rightarrow, \sim\rangle$, where $\sim$ is a symbol for the strong negation connective, containing axioms of positive logic and the following axioms for the strong negation:

$\left(1^{\sim}\right) \sim(p \vee q) \leftrightarrow(\sim p \wedge \sim q)$

$\left(2^{\sim}\right) \sim(p \wedge q) \leftrightarrow(\sim p \vee \sim q)$

$\left(3^{\sim}\right) \sim(p \rightarrow q) \leftrightarrow(p \wedge \sim q)$

$\left(4^{\sim}\right) \sim \sim p \leftrightarrow p$

Explosive Nelson's logic N3 is the least logic in the language $\mathcal{L}^{\sim}$ containing axioms of the logic N4 and the Duns Scottus law for strong negation: $\mathbf{N 3}=\mathbf{N} 4+\{\sim p \rightarrow(p \rightarrow q)\}$.

The logic $\mathbf{N} 4^{\perp}$ is defined in the language $\mathcal{L}^{*}=\mathcal{L}^{\sim} \cup\{\perp\}$ via axioms of $\mathbf{N} 4$ and the following axioms for the absurdity constant:

$$
\perp \rightarrow p, \sim \perp
$$

Notice that if we put $\perp:=\sim(p \rightarrow p)$, then

$$
\mathbf{N 3} \vdash \perp \rightarrow p, \sim \perp \text {. }
$$

Due to this reason we can consider $\mathbf{N} 3$ as a logic in the language $\mathcal{L}^{*}$ extending $\mathbf{N} 4^{\perp}$. Two other important extensions of the logic $\mathbf{N} 4^{\perp}$ are defined as follows:

$$
\mathbf{N} 4^{\mathrm{N}}=\mathbf{N} 4^{\perp}+\{\neg \neg(p \vee \sim p)\}, \quad \mathbf{N} 4^{\circ}=\mathbf{N} 3+\{\neg \neg(p \vee \sim p)\} .
$$

For a set $S \subseteq$ Prop, we denote by $\mathrm{Fm}^{+}(S)\left(F m^{\perp}(S), F m^{\sim}(S), F m^{*}(S)\right)$ the set of formulas of the language $\mathcal{L}^{+}\left(\mathcal{L}^{\perp}, \mathcal{L}^{\sim}, \mathcal{L}^{*}\right)$ with variables from $S$. We write $\mathrm{Fm}^{+}\left(\mathrm{Fm}^{\perp}\right.$, etc. $)$ instead of $\mathrm{Fm}^{+}$(Prop) $\left(\mathrm{Fm}^{\perp}\right.$ (Prop), etc.). For a set $F$ of formulas, we put $\sim F=\{\sim \varphi \mid \varphi \in F\}$. We denote by $\operatorname{Lit}(S)$ the set of literals from variables of $S, \operatorname{Lit}(S)=S \cup \sim S$. We write $L i t$ instead of Lit(Prop). Put $P_{n}=\left\{p_{1}, \ldots, p_{n}\right\}$ and $\operatorname{Lit}_{n}=\operatorname{Lit}\left(P_{n}\right)$.

Recall that $\mathbf{I P C}^{+}$coincides with the positive fragment of logics IPC, N3, and N4, i.e.,

$$
\mathbf{I P C}^{+}=\mathbf{I P C} \cap F m^{+}=\mathbf{N} 3 \cap F m^{+}=\mathbf{N} 4 \cap F m^{+} .
$$

At the same time, IPC is the $\sim$-free fragment of $\mathbf{N} 4^{\perp}, \mathbf{I P C}=\mathbf{N} \mathbf{4}^{\perp} \cap F m^{\perp}$.

A Kripke style semantics for Nelson's logic can be defined in a similar way to that for intuitionistic logic with the exception that valuations are defined on sets of literals.

A frame is a pair $\mathcal{W}=\langle W, \leq\rangle$, where $W$ is a non-empty set (of possible worlds), $\leq$ is a partial order on $W$. A subset $R$ of $W$ is called a cone of $\mathcal{W}$ (cone w.r.t. $\leq$ ) if it is upward closed w.r.t. $\leq$, i.e., for every $x \in R$ and $y \in W$, if $x \leq y$, then $y \in R$. We say that the cone $R$ is sharp if there is an element $a \in W$ such that $R=\{b \in W \mid a \leq b\}$. In this case we will use the denotation $R=[a]$ and $\langle a\rangle=[a] \backslash\{a\}$. For a subset $U$ of $W$, we denote $U \downarrow=\{x \in W \mid x \leq y$ for some $y \in U\}$.

An N4-model $\mathcal{M}=\langle W, \leq, V\rangle=\langle\mathcal{W}, V\rangle$ is a frame $\mathcal{W}$ augmented with a valuation $V: \operatorname{Lit}(S) \rightarrow 2^{W}$ such that $S$ is some set of propositional variables and $V(p)$ and $V(\sim p)$ are cones w.r.t. $\leq$ for all $p \in S$. In this case we say 
that $\mathcal{M}$ is a model over $\mathcal{W}$. We say that $\mathcal{M}$ is an N3-model if additionally $V(p) \cap V(\sim p)=\varnothing$ for all $p \in S$.

An N4-model $\mathcal{M}=\langle W, \leq, V\rangle$ with $V: \operatorname{Lit}(S) \rightarrow 2^{W}$ is said to be an $\mathbf{N} 4^{\mathrm{N}}$-model if the equality $(V(p) \cup V(\sim p)) \downarrow=W$ holds for all $p \in S$. We say that an $\mathbf{N} 3$-model $\mathcal{M}=\langle W, \leq, V\rangle$ is an $\mathbf{N} 4^{\circ}$-model if it is simultaneously an $\mathrm{N} 4^{\mathrm{N}}$-model.

The validity of formulas from $F m^{*}(S)$ at worlds of the model $\mathcal{M}$ is defined by induction. For $p \in S, \varphi, \psi \in F m^{*}(S)$ and $x \in W$ we put:

- $\mathcal{M}, x \Vdash p$ iff $x \in V(p), \quad \mathcal{M}, x \Vdash \sim p$ iff $x \in V(\sim p)$;

- $\mathcal{M}, x \Vdash \varphi \wedge \psi$ iff $\mathcal{M}, x \Vdash \varphi$ and $\mathcal{M}, x \Vdash \psi$;

- $\mathcal{M}, x \Vdash \varphi \vee \psi$ iff $\mathcal{M}, x \Vdash \varphi$ or $\mathcal{M}, x \Vdash \psi$;

- $\mathcal{M}, x \Vdash \varphi \rightarrow \psi$ iff $\forall y \geq x(\mathcal{M}, x \not \models \varphi$ or $\mathcal{M}, x \Vdash \psi)$;

- $\mathcal{M}, x \nVdash \perp, \quad \mathcal{M}, x \Vdash \sim \perp$;

- $\mathcal{M}, x \Vdash \sim(\varphi \wedge \psi)$ iff $\mathcal{M}, x \Vdash \sim \varphi$ or $\mathcal{M}, x \Vdash \sim \psi$;

- $\mathcal{M}, x \Vdash \sim(\varphi \vee \psi)$ iff $\mathcal{M}, x \Vdash \sim \varphi$ and $\mathcal{M}, x \Vdash \sim \psi$;

- $\mathcal{M}, x \Vdash \sim(\varphi \rightarrow \psi)$ iff $\mathcal{M}, x \Vdash \varphi$ and $\mathcal{M}, x \Vdash \sim \psi$;

- $\mathcal{M}, x \Vdash \sim \sim \varphi$ iff $\mathcal{M}, x \Vdash \varphi$.

If $\mathcal{M}, x \Vdash \varphi$ and $x \leq y$, then $\mathcal{M}, y \Vdash \varphi$. If $\mathcal{M}$ is an N3-model, then for every formula $\varphi$ and worlds $x$ we have $\mathcal{M}, x \forall \varphi \wedge \sim \varphi$.

A formula $\varphi$ is valid in $\mathcal{M}, \mathcal{M} \Vdash \varphi$, if $\mathcal{M}, x \Vdash \varphi$ for all $x \in W$. A formula $\varphi$ is valid in $\mathcal{W}, \mathcal{W} \Vdash \varphi$, if $\mathcal{M} \Vdash \varphi$ for all models $\mathcal{M}$ over $\mathcal{W}$. For a set of formulas $\Gamma$, we write $\mathcal{M}, x \Vdash \Gamma(\mathcal{M} \Vdash \Gamma)$ if $\mathcal{M}, x \Vdash \psi(\mathcal{M} \Vdash \psi)$ for all $\psi \in \Gamma$.

The semantics presented above is different from a more traditional approach (see, eg., [40]), which assumes the presence of two valuations $V^{+}$: $S \rightarrow 2^{W}$ and $V^{-}: S \rightarrow 2^{W}$ and two forcing relations $\models^{+}$and $\models^{-}$between worlds and formulas. The second relation is defined for variables as follows:

$$
x \models^{-} p \text { iff } x \in V^{-}(p) .
$$

The inductive steps for $\models^{-}$-definition repeat steps for strongly negated formulas in the above definition. The equivalence of two kinds of semantics is more or less obvious.

We often consider different models over the same frame $\mathcal{W}=\langle W, \leq\rangle$, therefore, it will be convenient to write $\mathcal{W}, x \Vdash_{V} \varphi$ instead of $\langle\mathcal{W}, V\rangle, x \Vdash \varphi$. We will use also shorter denotation $x \Vdash_{V} \varphi$ if it does not lead to a confusion.

If we have two models $\mathcal{M}_{1}=\left\langle\mathcal{W}, V_{1}\right\rangle$ and $\mathcal{M}_{2}=\left\langle\mathcal{W}, V_{2}\right\rangle$ over the same frame, we say that $\mathcal{M}_{1}$ and $\mathcal{M}_{2}\left(V_{1}\right.$ and $\left.V_{2}\right)$ agree on a set $S \subseteq \operatorname{Dom}\left(V_{1}\right) \cap$ $\operatorname{Dom}\left(V_{2}\right)$ if $V_{1} \uparrow \operatorname{Lit}(S)=V_{2} \uparrow \operatorname{Lit}(S)$.

If $\mathcal{M}=\langle W, \leq, V\rangle=\langle\mathcal{W}, V\rangle$ is a model and $X$ is a cone of $\mathcal{M}$, then $\mathcal{M}^{X}=\left\langle X, \leq \cap X^{2}, V^{X}\right\rangle$, where $V^{X}(\alpha)=V(\alpha) \cap X$ for every literal $\alpha$ from the domain of $V$, is a submodel of $\mathcal{M}$ generated by $X$. A model $\mathcal{M}_{1}$ is a generated submodel of $\mathcal{M}$ if $\mathcal{M}_{1}=\mathcal{M}^{X}$ for some cone $X$ of $\mathcal{W}$. It is known that for every formula $\varphi$ and $x \in X, x \Vdash_{V} \varphi$ iff $x \Vdash_{V^{X}} \varphi$.

Let $\mathcal{M}=\langle M, \leq, V\rangle$ be a model such that $V: \operatorname{Lit}(S) \rightarrow 2^{M}$ and $X$ is a cone of $\mathcal{M}$. The cone $X$ is said to be $S$-definable $\left(S^{*}\right.$-definable) if there is a 
formula $\varphi \in F m^{\sim}(S)\left(\varphi \in F m^{*}(S)\right)$ such that $\forall x \in M(\mathcal{M}, x \Vdash \varphi \Leftrightarrow x \in$ $X)$. A valuation $V_{1}: \operatorname{Lit}\left(S_{1}\right) \rightarrow 2^{M}$ is said to be $(*-)$ definable in $\mathcal{M}$ if for every $p \in S_{1}$, the cones $V_{1}(p)$ and $V_{1}(\sim p)$ are $S$-definable ( $S^{*}$-definable).

In what follows we assume an agreement that if a model is denoted by a calligraphic latter, its set of worlds is denoted by the same italic letter, $\mathcal{M}=\langle M, \leq, V\rangle$.

It is known that $\mathbf{N} \mathbf{4}$ and $\mathbf{N} \mathbf{4}^{\perp}$ are strongly complete w.r.t. the class of all frames and complete w.r.t. the class of all finite frames. The logic N3 is strongly complete w.r.t. the class of all N3-models and complete w.r.t. the class of all finite $\mathbf{N} 3$-models. In [17], it was proved that the logic $\mathbf{N} 4^{\mathrm{N}}\left(\mathbf{N} 4^{\circ}\right)$ is strongly complete w.r.t. the class of all $\mathbf{N} 4^{\mathrm{N}}$-models $\left(\mathbf{N} 4^{\circ}\right.$-models) and complete w.r.t. the class of all finite $\mathbf{N} 4^{\mathrm{N}}$-models $\left(\mathbf{N} 4^{\circ}\right.$-models).

If a model $\mathcal{M}=\langle W, \leq, V\rangle$ with $V: \operatorname{Lit}(S) \rightarrow 2^{W}$ is finite, then every world of this model lies under some maximal world. Clearly, for finite models the defining condition of $\mathbf{N} 4^{\mathrm{N}}$-models can be replaced by the following: every maximal world $x \in W$ belongs to $V(p) \cup V(\sim p)$ for all $p \in S$. In other worlds,

$$
x \Vdash p \text { or } x \Vdash \sim p \text { for all } p \in S .
$$

The latter condition is equivalent to

$$
x \Vdash \varphi \text { or } x \Vdash \sim \varphi \text { for all } \varphi \in F m^{*}(S) .
$$

Thus, finite $\mathbf{N} 4^{\mathrm{N}}$-models are $\mathbf{N} 4$-models, where all maximal worlds are complete w.r.t. $\sim$. We know that $\mathbf{N 3 - m o d e l s ~ a r e ~} \mathbf{N} 4$-models, where all worlds are consistent w.r.t. $\sim$. Consequently, finite $\mathbf{N} 4^{\circ}$-models are exactly finite $\mathbf{N 3 -}$ models, where all maximal worlds are classical, i.e. consistent and complete w.r.t. $\sim$.

For a model $\mathcal{M}=\langle M, \leq, V\rangle$ with $V: \operatorname{Lit}(S) \rightarrow 2^{W}$ and $x \in M$, we put $\operatorname{Lit}_{\mathcal{M}}(x)=\{\alpha \in \operatorname{Lit}(S) \mid \mathcal{M}, x \Vdash \alpha\}$, i.e., $\operatorname{Lit}_{\mathcal{M}}(x)$ is the set of literals valid at the world $x$ of the model $\mathcal{M}$. In denotation $\operatorname{Lit}_{\mathcal{M}}(x)$ we omit the lower index, if it is clear from the context, which model we mean.

We will need a small refinement of the finite model property for Nelson's logics. Assume that an N4-model $\mathcal{M}=\langle M, \leq, V\rangle$ with $V: \operatorname{Lit}(S) \rightarrow 2^{M}$ has an element $x$ such that $\mathcal{M}, x \Vdash \operatorname{Lit}(S)$. It is easy to see that in this case $\mathcal{M}, x \Vdash F m^{\sim}(S)$ (of course, $\mathcal{M}, x \Downarrow \perp$ ). The set of all elements with this property form a cone w.r.t. $\leq$ which we denote $A l l$. Let us delete this cone from $\mathcal{M}$, that is we pass from $\mathcal{M}$ to the new model $\mathcal{M}^{\prime}=\left\langle M \backslash A l l, \leq^{\prime}, V^{\prime}\right\rangle$, where $\leq^{\prime}=\leq \uparrow_{M \backslash A l l}$ and $V^{\prime}(\alpha)=V(\alpha) \backslash A l l$ for $\alpha \in \operatorname{Lit}(S)$. An easy induction on the complexity of formulas shows that

$$
\mathcal{M}, x \Vdash \varphi \quad \Leftrightarrow \quad \mathcal{M}^{\prime}, x \Vdash \varphi
$$

for all $x \notin A l l$ and $\varphi \in F m^{*}(S)$.

For $x, y \in M$, the world $y$ is said to be a duplication of $x$ if $x$ is the only immediate successor of $y$ w.r.t. $\leq$ and $\operatorname{Lit}_{\mathcal{M}}(x)=\operatorname{Lit}_{\mathcal{M}}(y)$. We say that an $\mathbf{N 4}$-model $\mathcal{M}$ has no duplications if there is no $y \in M$ which is a duplication of another element of $M$. We say that $x$ and $y$ are twins, if they are incomparable, have the same successors w.r.t. $\leq$, and $\operatorname{Lit}_{\mathcal{M}}(x)=\operatorname{Lit}_{\mathcal{M}}(y)$. 
If $y$ is a duplication of $x$, or $x$ and $y$ are twins, it follows by an easy induction on the length of formulas that for all $\varphi$ we have $x \Vdash_{V} \varphi$ iff $y \Vdash_{V}$ $\varphi$. Obviously, any filtration of an $\mathbf{N} 4$-model has no duplications and twins. Therefore, we may conclude that every formula $\varphi \notin \mathbf{N} 4\left(\varphi \notin \mathbf{N} 4^{\perp}\right)$ is refuted on a finite $\mathbf{N 4}$-model $\mathcal{M}$ which has no duplications and twins, and has no world $x$ such that $\mathcal{M}, x \vdash \operatorname{Lit}(S)$. In a similar way, every formula $\varphi \notin \mathbf{N 3}$ $\left(\mathbf{N} 4^{\mathrm{N}}, \mathbf{N} 4^{\circ}\right)$ is refuted on a finite $\mathbf{N} 3-\left(\mathbf{N} 4^{\mathrm{N}}-, \mathbf{N} 4^{\circ}-\right)$ model with the same properties.

Concluding the section we discuss normal forms of formulas with strong negation and embeddings of Nelson's logics into positive and intuitionistic logics.

We say that $\varphi \in F m^{\sim}(S)\left(\varphi \in F m^{*}(S)\right)$ is a negative normal form $(n n f)$ if the strong negation connective occur in $\varphi$ only in front of atomic formulas. Since the logics $\mathbf{N} 4$ and $\mathbf{N} 4^{\perp}$ contain all axioms of $\mathbf{I P C} \mathbf{C}^{+}$one can prove that these logic are closed under the positive replacement rule:

$$
\frac{\varphi \leftrightarrow \psi}{\chi(\varphi) \leftrightarrow \chi(\psi)},
$$

where $\chi(p)$ is a $\sim$-free formula.

However, these logics are not closed under the usual replacement rule. Indeed, we have $\sim(p \rightarrow q) \leftrightarrow(p \wedge \sim q) \in \mathbf{N} 4$. Assume that $\sim \sim(p \rightarrow q) \leftrightarrow \sim$ $(p \wedge \sim q) \in \mathbf{N 4}$. The left side of this equivalence is equivalent to $p \rightarrow q$ by axiom $\left(4^{\sim}\right)$, the right side is equivalent to $\sim p \vee q$ by $\left(2^{\sim}\right),\left(4^{\sim}\right)$, and the positive replacement rule. Thus, $(p \rightarrow q) \leftrightarrow(\sim p \vee q) \in \mathbf{N} 4$. This fact obviously contradicts to semantical characterization of $\mathbf{N 4}$.

The fact that Nelson's logics are closed under the positive replacement rules and the strong negation axioms allow us to prove that every formula can be reduced to a negative normal form. More precisely, let $L \in$ $\left\{\mathbf{N 3}, \mathbf{N} 4, \mathbf{N 4}^{\perp}\right\}$. For every formula $\varphi$, there is a $n n f \psi$ such that

$$
\varphi \leftrightarrow \psi \in L .
$$

In what follows we assume that we have fixed an algorithm assigning to a formula $\varphi$ its negative normal form $\varphi^{\natural}$.

$$
\begin{aligned}
& \text { Let } \varphi(\bar{p})=\varphi\left(p_{1}, \ldots, p_{n}\right) \in F m^{\sim}\left(F m^{*}\right), \\
& \qquad \varphi^{\natural}=\varphi(\bar{p}, \sim \bar{p})=\varphi^{\prime}\left(p_{1}, \ldots, p_{n}, \sim p_{1}, \ldots, \sim p_{n}\right),
\end{aligned}
$$

where $\varphi^{\prime} \in F m^{+}$(resp., $\varphi^{\prime} \in F m^{\perp}$ ).

Put

$$
\begin{gathered}
\varphi^{4}:=\varphi^{\prime}\left(\bar{p}, \bar{p}^{\dagger}\right)=\varphi^{\prime}\left(p_{1}, \ldots, p_{n}, p_{1}^{\dagger}, \ldots, p_{n}^{\dagger}\right) ; \\
\varphi^{\mathbf{3}}:=\left(\bigwedge_{i=1}^{n} \neg\left(p_{i} \wedge p_{i}^{\dagger}\right)\right) \rightarrow \varphi^{\mathbf{4}} ;
\end{gathered}
$$

We have just defined embeddings of Nelson's logic into positive intuitionistic and intuitionistic logics.

Theorem 2.1. 1 . For every $\varphi \in F m^{\sim}$, the following equivalence holds:

$$
\varphi \in \mathbf{N} 4 \text { iff } \varphi^{\mathbf{4}} \in \mathbf{I P C}^{+} \text {. }
$$


2. For every $\varphi \in F m^{\sim}$, the following equivalences hold:

$$
\begin{gathered}
\varphi \in \mathbf{N} 4^{\perp} \quad \text { iff } \varphi^{\mathbf{4}} \in \mathbf{I P C} \\
\varphi \in \mathbf{N} 3 \text { iff } \varphi^{\mathbf{3}} \in \mathbf{I P C}
\end{gathered}
$$

The statement for N3 was originally proved by Vorob'ev [38].

\section{3. n-Characterizing models for Nelson's logics}

An N4-model $\mathcal{M}=\langle W, \leq, V\rangle$ with $V:$ Lit $_{n} \rightarrow 2^{W}$ is said to be $n$-characterizing for a logic $L$ extending $\mathbf{N} 4\left(\mathbf{N 4}^{\perp}\right)$ iff for every $\varphi \in F m^{\sim}\left(P_{n}\right)\left(\varphi \in F m^{*}\left(P_{n}\right)\right)$, we have $\varphi \in L$ iff $\mathcal{M} \Vdash \varphi$.

The semantics of Nelson's logics treats the literals $\sim p$ in the same way as propositional variables. Due to this reason the construction of $n$ characterizing model for $\mathbf{N} 4\left(\mathbf{N} \mathbf{4}^{\perp}\right)$ is very similar to the construction of $2 n$-characterizing model for $\mathbf{I P C}^{+}$(IPC) from [24]. To avoid confusions we provide however the construction with all details.

For an $\mathbf{N} 4$-model $\mathcal{M}=\langle W, \leq, V\rangle$, we denote by $S l_{i}(\mathcal{M})$ the set of $x \in W$ with depth $i$ w.r.t. $\leq$. Put $S_{i}(\mathcal{M})=\bigcup_{1 \leq j \leq i} S l_{j}(\mathcal{M})$.

Now we construct an $n$-characterizing model for the logic $\mathbf{N} 4$ as a join of an ascending chain of $\mathbf{N} 4$-models $\mathcal{N}_{k}=\left\langle N_{k}, \leq_{k}, V_{k}\right\rangle, V_{k}:$ Lit $_{n} \rightarrow 2^{I_{k}}$, $k \in \omega$.

We start with a model $\mathcal{N}_{1}=\left\langle N_{1}, \leq_{1}, V_{1}\right\rangle$ such that $\left\langle N_{1}, \leq_{1}\right\rangle$ is an antichain, and the valuation $V_{1}$ is chosen so that

$$
\begin{gathered}
\forall x, y \in N_{1}\left(x \neq y \Rightarrow \text { Lit }_{\mathcal{N}_{1}}(x) \neq \text { Lit }_{\mathcal{N}_{1}}(y)\right), \\
\left\{\text { Lit }_{\mathcal{N}_{1}}(x) \mid x \in N_{1}\right\}=2^{\text {Lit }_{n}} \backslash\left\{\text { Lit }_{n}\right\} .
\end{gathered}
$$

Assume that the model $\mathcal{N}_{k}=\left\langle N_{k}, \leq_{k}, V_{k}\right\rangle$ has been constructed and define $\mathcal{N}_{k+1}$. Let $A C_{k}$ be the set of all antichains of the model $\mathcal{N}_{k}$ which contain at least one element of depth $k$. For each $\Delta \in A C_{k}$, we consider new elements $\Delta_{j}$ and sets $\operatorname{Lit}\left(\Delta_{j}\right) \subseteq \operatorname{Lit}_{n}$ indexed by elements of such a set $X^{\Delta}$ that

$$
\forall j_{1}, j_{2} \in X^{\Delta}\left(j_{1} \neq j_{2} \Rightarrow \operatorname{Lit}\left(\Delta_{j_{1}}\right) \neq \operatorname{Lit}\left(\Delta_{j_{2}}\right)\right)
$$

if $\|\Delta\| \geq 2$, then

$$
\left\{\operatorname{Lit}\left(\Delta_{j}\right) \mid j \in X^{\Delta}\right\}=\left\{Y \mid Y \subseteq \bigcap\left\{\operatorname{Lit}_{\mathcal{N}_{k}}(x) \mid x \in \Delta\right\}\right\}
$$

if $\Delta=\{b\}$, then

$$
\left\{\operatorname{Lit}\left(\Delta_{j}\right) \mid j \in X^{\Delta}\right\}=\left\{Y \mid Y \varsubsetneqq \operatorname{Lit}_{\mathcal{N}_{k}}(b)\right\} ;
$$

Let $N_{k+1}=N_{k} \cup\left\{\Delta_{j} \mid \Delta \in A C_{k}, j \in X^{\Delta}\right\}$. For $\Delta_{j} \in N_{k+1} \backslash N_{k}$ and $x \in N_{k}$, we put

$$
\Delta_{j} \sqsubset x \text { iff }(x \in \Delta) \vee \exists y \in \Delta\left(y \leq_{k} x\right) .
$$

Define the relation $\leq_{k+1}$ on $N_{k+1}$ by putting

$$
\leq_{k+1}=\leq_{k} \cup \sqsubset \cup I d_{N_{k+1}} .
$$


Obviously, we have defined a partial ordering $N_{k+1}$. Finally, we define the valuation:

$$
V_{k+1}(\alpha)=V_{k}(\alpha) \cup\left\{\Delta_{j} \mid \Delta \in A C_{k}, j \in X^{\Delta}, \alpha \in \operatorname{Lit}\left(\Delta_{j}\right)\right\},
$$

where $\alpha \in$ Lit $_{n}$. It is easy to see that $V_{k+1}(\alpha)$ is a cone w.r.t. $\leq_{k+1}$.

Thus, the N4-model $\mathcal{N}_{k+1}=\left\langle N_{k+1}, \leq_{k+1}, V_{k+1}\right\rangle$ is completely defined. It is clear that $N_{k}$ is a cone w.r.t. $\leq_{k+1}$ and $N_{k}=S_{k}\left(\mathcal{N}_{k+1}\right)$, respectively the model $\mathcal{N}_{k}$ is a generated submodel of $\mathcal{N}_{k+1}$.

The N4-model $\mathcal{N}(n)=\left\langle N(n), \leq, V^{(n)}\right\rangle$ is defined so that

$$
N(n)=\bigcup_{k>0} N_{k}, \leq=\bigcup_{k>0} \leq_{k}, V^{(n)}(\alpha)=\bigcup_{k>0} V_{k}(\alpha) .
$$

Proposition 3.1. The model $\mathcal{N}(n)$ is $n$-characterizing for the logics $\mathbf{N} 4$ and N4 ${ }^{\perp}$.

Proof. Since $\mathcal{N}(n)$ is an $\mathbf{N} 4$-model, we have $\mathcal{N}(n) \Vdash \varphi$ for all $\varphi \in \mathbf{N} 4 \cap$ $F m^{\sim}\left(P_{n}\right)$. Assume that $\varphi \in F m^{\sim}\left(P_{n}\right) \backslash \mathbf{N} 4$, then there exists a finite N4-model $\mathcal{M}$ refuting $\varphi$, which has no duplications and twins, and has no worlds $x$ such that $\mathcal{M}, x \Vdash L_{i} t_{n}$. Due to this reason for every maximal element $x$ of $\mathcal{M}$ one can find a maximal element $y$ of $\mathcal{N}(n)$ such that $\operatorname{Lit}_{\mathcal{M}}(x)=\operatorname{Lit}_{\mathcal{N}(n)}(y)$. Identifying maximal elements of $\mathcal{M}$ with respective maximal elements of $\mathcal{N}(n)$ we obtain the model $\mathcal{M}_{1}$, i.e., we replace the maximal elements from $\mathcal{M}$ by respective maximal elements of $\mathcal{N}(n)$. Thus, $S_{1}\left(\mathcal{M}_{1}\right)$ is a cone of $\mathcal{N}(n)$. Assume that we have already constructed a model $\mathcal{M}_{k}$ such that $S_{k}\left(\mathcal{M}_{k}\right)$ is a cone of $\mathcal{N}(n)$, the depth of $\mathcal{M}_{k}$ is not greater then the depth of $\mathcal{M}$, and $\mathcal{M}_{k} \nVdash \varphi$. Let us construct a model $\mathcal{M}_{k+1}$ with similar properties.

Since $\mathcal{M}$ has no duplications, for every element $x \in S l_{k+1}\left(\mathcal{M}_{k}\right)$, there is an element $a \in N_{k+1}$ such that

$$
\operatorname{Lit}_{\mathcal{M}_{k}}(x)=\operatorname{Lit}_{\mathcal{N}(n)}(a) \text { and }\left\{y \in M_{k} \mid x<y\right\}=\{y \in N(n) \mid a<y\} \text {. }
$$

Since $\mathcal{M}$ has no twins, there is only one element $x \in S l_{k+1}\left(\mathcal{M}_{k}\right)$ satisfying (1), and we identify this element with the element $a$ of $\mathcal{N}(n)$. In this way we obtain the model $\mathcal{M}_{k+1}$. It is not hard to see that $\mathcal{M}_{k+1} \nVdash \varphi$. Clearly, $S_{k+1}\left(\mathcal{M}_{k+1}\right)$ is a cone of $N(n)$, and the depth of $\mathcal{M}_{k+1}$ is equal to the depth of $\mathcal{M}_{k}$. Since $\mathcal{M}$ is finite, after a finite number of steps we obtain a model $\mathcal{M}_{m}$ such that it is a cone of $\mathcal{N}(n)$ and $\mathcal{M}_{m} \nVdash \varphi$. Consequently, $\mathcal{N}(n) \nVdash \varphi$.

To prove that $\mathcal{N}(n)$ is $n$-characterizing for $\mathbf{N} 4^{\perp}$ let us take a formula $\varphi \in F m^{*}\left(P_{n}\right)$ such that $\varphi \notin \mathbf{N} 4^{\perp}$. This formula is refutable on a finite $\mathbf{N} 4$ model $\mathcal{M}$, which has no duplications and twins, and has no worlds $x$ such that $\mathcal{M}, x \Vdash L_{i} t_{n}$. We just proved that every such model can be embedded into $\mathcal{N}(n)$ as a cone. Consequently, $\varphi$ is refutable on $\mathcal{N}(n)$ too.

Proposition 3.2. Every sharp cone of the model $\mathcal{N}(n)$ is $P_{n}$-definable. 
Proof. We construct defining formulas for sharp cones $[x]$ using induction on the depth of $x$.

For $x \in S_{1}(\mathcal{N}(n))$, put

$$
f(x):=\bigwedge \operatorname{Lit}_{\mathcal{N}(n)}(x) \wedge\left(\bigvee\left(\operatorname{Lit}_{n} \backslash \operatorname{Lit}_{\mathcal{N}(n)}(x)\right) \rightarrow \bigwedge \operatorname{Lit}_{n}\right)
$$

It is clear that $\mathcal{N}(n), y \Vdash f(x)$ iff $y=x$.

Assume that for every $x \in S_{m}(\mathcal{N}(n))$ we have constructed the formula $f(x)$ that distinguishes the sharp cone $[x]$ from other elements of $\mathcal{N}(n)$. For all $x \in S l_{m+1}(\mathcal{N}(n))$, define

$$
g(x):=\bigwedge\left\{f(c) \rightarrow \bigvee_{x<b} f(b) \mid x \not \leq c, c \in S_{m}(\mathcal{N}(n))\right\} .
$$

It is clear that for every $y \in S_{m+1}(\mathcal{N}(n))$, we have

$$
\mathcal{N}(n), y \Vdash g(x) \Leftrightarrow\langle y\rangle \cap N_{m} \subseteq\langle x\rangle \cap N_{m} .
$$

Further, we let

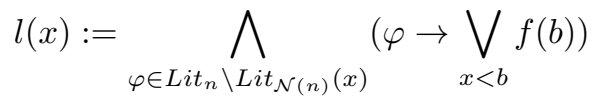

and

$$
h(x):=\bigwedge\left\{g(c) \rightarrow \bigvee_{r \in S_{m}(\mathcal{N}(n))} f(r) \mid c \in S l_{m+1}(\mathcal{N}(n)),\langle c\rangle \varsubsetneqq\langle x\rangle\right\}
$$

for all $x \in S l_{m+1}(\mathcal{N}(n))$. Finally,

$$
f(x):=\bigwedge \operatorname{Lit}_{\mathcal{N}(n)}(x) \wedge g(x) \wedge l(x) \wedge h(x) .
$$

Consider some $a \in S l_{m+1}(\mathcal{N}(n))$ and prove that $f(a)$ is the desired formula, i.e., that $f(a)$ defines $[a]$. It is easy to see that $\mathcal{N}(n), a \Vdash f(a)$. Consider an element $d \in S l_{m+1}(\mathcal{N}(n))$ with $\langle d\rangle \neq\langle a\rangle$. Then either $\langle d\rangle \nsubseteq$ $\langle a\rangle$ or $\langle d\rangle \varsubsetneqq\langle a\rangle$. If $\langle d\rangle \nsubseteq\langle a\rangle$, then $\mathcal{N}(n), d \nVdash g(a)$ by $(2)$. If $\langle d\rangle \varsubsetneqq\langle a\rangle$, then $\mathcal{N}(n), d \Vdash g(d)$, but $\mathcal{N}(n), d \nVdash \bigvee_{r \in S_{m}(\mathcal{N}(n))} f(r)$ since $d \notin S l_{m}(\mathcal{N}(n))$. Consequently, $\mathcal{N}(n), d \nVdash h(a)$.

Let $d \in S l_{m+1}(\mathcal{N}(n)),\langle d\rangle=\langle a\rangle$, and $d \neq a$. If $\operatorname{Lit}_{\mathcal{N}(n)}(a) \nsubseteq \operatorname{Lit}_{\mathcal{N}(n)}(d)$, then $\mathcal{N}(n), d \nVdash \wedge \operatorname{Lit}_{\mathcal{N}(n)}(a)$. Assume that $\operatorname{Lit}_{\mathcal{N}(n)}(a) \varsubsetneqq \operatorname{Lit}_{\mathcal{N}(n)}(d)$ and $\varphi \in \operatorname{Lit}_{\mathcal{N}(n)}(d) \backslash \operatorname{Lit}_{\mathcal{N}(n)}(a)$. We have $\mathcal{N}(n), d \Vdash \varphi$ and $\mathcal{N}(n), d \nVdash \bigvee_{a<b} f(b)$, whence $\mathcal{N}(n), d \nVdash l(a)$. We have thus proved that

$$
\forall d \in S l_{m+1}(\mathcal{N}(n))(d \neq a \Rightarrow \mathcal{N}(n), d \nVdash f(a)) .
$$

Assume now that $r \in S_{m+2+k}(\mathcal{N}(n))$ and $\mathcal{N}(n), r \Vdash f(a)$. By (3) we have $[r] \cap S l_{m+1}(\mathcal{N}(n))=\{a\}$ and there exists $d \in S l_{m+2}(\mathcal{N}(n))$ with $r \leq$ $d<a$.

Let, first, $\langle d\rangle=[a]$. Then, by construction of $N(n)$ we have $\operatorname{Lit}_{\mathcal{N}(n)}(d) \varsubsetneqq$ $\operatorname{Lit}_{\mathcal{N}(n)}(a)$. Consequently, $\mathcal{N}(n), r \nVdash \wedge \operatorname{Lit}_{\mathcal{N}(n)}(a)$ and $\mathcal{N}(n), r \nVdash f(a)$.

Let now, $\langle d\rangle \neq[a]$. Then there is $b, b \in S_{m}(\mathcal{N}(n))$, where $d<b, b \notin\langle a\rangle$. In this case we get $\mathcal{N}(n), d \nVdash g(a)$ and consequently, $\mathcal{N}(n), r \nVdash f(a)$. 
Finally, suppose that $c \in S_{m}(\mathcal{N}(n)), a \not \leq c$, and $\mathcal{N}(n), c \Vdash f(a)$. We have also $\mathcal{N}(n), c \Vdash f(c)$. From $\mathcal{N}(n), c \Vdash g(a)$ we obtain $\mathcal{N}(n), c \Vdash f(b)$ for some $b>a$. Consequently, $a<c$, which contradicts the assumption $a \not \leq c$.

It remains to construct an $n$-characteristic models $\mathcal{N}^{\mathbf{3}}(n), \mathcal{N}^{\mathrm{N}}(n)$, and $\mathcal{N}^{\circ}(n)$ for the logics $\mathbf{N} 3, \mathbf{N} 4^{\mathrm{N}}$, and respectively $\mathbf{N} 4^{\circ}$. We define them as a generated submodels of $\mathcal{N}(n)$ with the following set of worlds:

$$
\begin{gathered}
N^{\mathbf{3}}(n)=\left\{x \in N(n) \mid \forall p \in P_{n} \forall y \geq x(y \Vdash p \wedge \sim p)\right\} ; \\
N^{\mathrm{N}}(n)=\left\{x \in N(n) \mid \forall p \in P_{n} \forall y \geq x\left(y \in S^{1}(\mathcal{N}(n)) \Rightarrow y \Vdash p \vee \sim p\right)\right\} ; \\
N^{\circ}(n)=\left\{x \in N^{\mathbf{3}}(n) \mid \forall p \in P_{n} \forall y \geq x\left(y \in S^{1}(\mathcal{N}(n)) \Rightarrow y \Vdash p \vee \sim p\right)\right\} .
\end{gathered}
$$

In other worlds, the model $\mathcal{N}^{\mathbf{3}}(n)$ consists of those worlds of $\mathcal{N}(n)$, which are consistent and have only consistent extensions; the model $\mathcal{N}^{\mathrm{N}}(n)$ consists of worlds with complete maximal extensions. Finally, $\mathcal{N}^{\circ}(n)$ consists of consistent worlds with classical maximal extensions.

Proposition 3.3. The model $\mathcal{N}^{3}(n)\left(\mathcal{N}^{\mathrm{N}}(n), \mathcal{N}^{\circ}(n)\right)$ is $n$-characterizing for the logic $\mathbf{N} 3\left(\mathbf{N} 4^{\mathrm{N}}, \mathbf{N} 4^{\circ}\right)$.

Proof. It is obvious that $\mathcal{N}^{\mathbf{3}}(n)$ is an $\mathbf{N} 3$-model, therefore, $\mathcal{N}^{\mathbf{3}}(n) \Vdash \varphi$ for all $\varphi \in \mathbf{N} 3 \cap F m^{*}\left(P_{n}\right)$. To complete the proof let us take a formula $\varphi \in F m^{*}\left(P_{n}\right)$ such that $\varphi \notin \mathbf{N} 3$. This formula is refutable on a finite $\mathbf{N 3}$-model $\mathcal{M}$, which has no duplications and twins, and has no worlds $x$ such that $\mathcal{M}, x \Vdash$ Lit $_{n}$. It is also an $\mathbf{N 4}$-model and we know that $\mathcal{M}$ can be embedded into $\mathcal{N}(n)$ as a cone. Since all worlds of the N3-model $\mathcal{M}$ are consistent, its image is contained in $\mathcal{N}^{\mathbf{3}}(n)$. Thus, $\mathcal{M}$ can be considered as a generated submodel of $\mathcal{N}^{\mathbf{3}}(n)$, and we may conclude that $\varphi$ is refutable on $\mathcal{N}^{\mathbf{3}}(n)$, i.e., $\mathcal{N}^{\mathbf{3}}(n)$ is $n$-characterizing for $\mathbf{N} 3$.

To prove that $\mathcal{N}^{\mathrm{N}}(n)$ is $n$-characterizing for $\mathbf{N} 4^{\mathrm{N}}$ we have to embed an arbitrary finite $\mathbf{N} 4^{\mathrm{N}}$-model $\mathcal{M}$ as cone into $\mathcal{N}^{\mathrm{N}}(n)$. Let us consider the image of $\mathcal{M}$ in $\mathcal{N}(n)$. We know that all maximal worlds of $\mathcal{M}$ are complete. It is obvious that the images of maximal complete worlds are maximal and complete as well. Thus, the image of $\mathcal{M}$ is a subset of $\mathbf{N} 4^{\mathrm{N}}$.

For $\mathcal{N}^{\circ}(n)$, the proof follows the same line.

All sharp cones of $\mathcal{N}^{\mathbf{3}}(n), \mathcal{N}^{\mathrm{N}}(n)$, and $\mathcal{N}^{\circ}(n)$ are $P_{n}$-definable, because they are cones of $\mathcal{N}(n)$.

\section{Admissibility and weak admissibility of inference rules in $\mathrm{N} 4$ and $\mathrm{N} 4^{\perp}$}

We recall that a rule of inference with parameters is an expression $r$ of the form

$$
\frac{\varphi_{1}(\bar{x}, \bar{p}), \ldots, \varphi_{n}(\bar{x}, \bar{p})}{\psi(\bar{x}, \bar{p})},
$$


where $\varphi_{i}$ and $\psi$ are formulas, $\bar{x}$ and $\bar{p}$ are tuples of propositional variables, $\bar{x}$ are variables of $r$ and $\bar{p}$ are parameters of $r$. The rule $r$ is said to be admissible in a logic $L$ extending $\mathbf{N} 4$ or $\mathbf{N} 4^{\perp}$ if for every tuple of formulas $\bar{\xi}$ in the respective language, we have $\psi(\bar{\xi}, \bar{p}) \in L$ whenever $\varphi_{1}(\bar{\xi}, \bar{p}), \ldots, \varphi_{n}(\bar{\xi}, \bar{p}) \in L$. Obviously, the rule $r$ is admissible in $L$ iff the rule

$$
\frac{\varphi_{1}(\bar{x}, \bar{p}) \wedge \ldots \wedge \varphi_{n}(\bar{x}, \bar{p})}{\psi(\bar{x}, \bar{p})}
$$

is admissible in $L$. Therefore, in what follows we consider only rules with one premiss. Further, since every formula is equivalent in $L$ to its negative normal form, the rule

$$
\frac{\varphi(\bar{x}, \bar{p})}{\psi(\bar{x}, \bar{p})}
$$

is admissible in a logic $L$ iff the rule

$$
\frac{\varphi^{\natural}(\bar{x}, \bar{p})}{\psi^{\natural}(\bar{x}, \bar{p})}
$$

is admissible in $L$. So, studying the admissibility problem in extensions of Nelson's logics it will be sufficient to consider rules of the form

$$
\frac{\varphi(\bar{x}, \sim \bar{x}, \bar{p}, \sim \bar{p})}{\psi(\bar{x}, \sim \bar{x}, \bar{p}, \sim \bar{p})},
$$

where $\varphi, \psi \in F m^{+}$if we consider $\mathbf{N} 4$-extensions, and $\varphi, \psi \in F m^{\perp}$ in case of $\mathbf{N} 4^{\perp}$-extensions.

First we notice that the admissibility problem for the logics $\mathbf{N} 4$ and $\mathbf{N} 4^{\perp}$ is equivalent to the following strengthening of admissibility problem for IPC $^{+}$and respectively IPC considered over the extended set of propositional variables Prop $\cup \operatorname{Prop}^{\dagger}$.

For $\varphi \in F m^{\perp}\left(\operatorname{Prop} \cup \operatorname{Prop}{ }^{\dagger}\right)$, we define its dual formula $\varphi^{d}$ as follows:

$$
\begin{aligned}
p_{i}^{d} & :=p_{i}^{\dagger} & \left(p_{i}^{\dagger}\right)^{d} & :=p_{i} \\
(\psi \wedge \chi)^{d} & :=\psi^{d} \vee \chi^{d} & (\psi \vee \chi)^{d} & :=\psi^{d} \wedge \chi^{d} \\
(\psi \rightarrow \chi)^{d} & :=\psi \wedge \chi^{d} & \perp^{d} & :=p_{0} \rightarrow p_{0}
\end{aligned}
$$

Let $\bar{x}$ and $\bar{y}$ denote disjoint tuples of propositional variables of the same length. For $\varphi(\bar{x}, \bar{y}, \bar{p}), \psi(\bar{x}, \bar{y}, \bar{p}) \in F m^{+}\left(\operatorname{Prop} \cup \operatorname{Prop}^{\dagger}\right)\left(F m^{\perp}\left(\operatorname{Prop} \cup \operatorname{Prop}^{\dagger}\right)\right)$ the rule

$$
\frac{\varphi(\bar{x}, \bar{y}, \bar{p})}{\psi(\bar{x}, \bar{y}, \bar{p})}
$$

is $d$-admissible $e^{1}$ in a logic $L$ extending IPC $^{+}$(IPC) if for every tuple $\bar{\xi}$ of formulas from $\mathrm{Fm}^{+}\left(\operatorname{Prop} \cup \operatorname{Prop}^{\dagger}\right)\left(F m^{\perp}\left(\operatorname{Prop} \cup \operatorname{Prop}{ }^{\dagger}\right)\right)$ we have $\psi\left(\bar{\xi}, \bar{\xi}^{d}, \bar{p}\right) \in$ $L$ whenever $\varphi\left(\bar{\xi}, \bar{\xi}^{d}, \bar{p}\right) \in L$. Here $\bar{\xi}^{d}$ denote a tuple of formulas dual to formulas from $\bar{\xi}$.

\footnotetext{
${ }^{1}$ The letter "d" is from "dependent", because there is a dependence between formulas substituted for variables $x_{i}$ and $y_{i}$. If $x_{i}$ is replaced by $\xi_{i}$, then $y_{i}$ must be replaced by its dual $\xi_{i}^{d}$.
} 
Proposition 4.1. Let $\varphi(\bar{x}, \bar{y}, \bar{p}, \bar{q}), \psi(\bar{x}, \bar{y}, \bar{p}, \bar{q}) \in F m^{+}(\operatorname{Prop})\left(F m^{\perp}(\operatorname{Prop})\right)$. The rule

$$
\frac{\varphi(\bar{x}, \sim \bar{x}, \bar{p}, \sim \bar{p})}{\psi(\bar{x}, \sim \bar{x}, \bar{p}, \sim \bar{p})}
$$

is admissible in $\mathbf{N} 4\left(\mathbf{N} 4^{\perp}\right)$ iff the rule

$$
\frac{\varphi\left(\bar{x}, \bar{y}, \bar{p}, \bar{p}^{d}\right)}{\psi\left(\bar{x}, \bar{y}, \bar{p}, \bar{p}^{d}\right)}
$$

with parameters $\bar{p}$ and $\bar{p}^{d}$ is d-admissible in $\mathbf{I P C}{ }^{+}$(IPC).

Proof. We only consider the case of $\mathbf{N} 4$ and $\mathbf{I P C}^{+}$.

Assume that $\bar{\xi}$ is such that $\varphi(\bar{\xi}, \sim \bar{\xi}, \bar{p}, \sim \bar{p}) \in \mathbf{N} 4$, but $\psi(\bar{\xi}, \sim \bar{\xi}, \bar{p}, \sim$ $\bar{p}) \notin \mathbf{N} 4$. According to Theorem 2.1 we conclude $(\varphi(\bar{\xi}, \sim \bar{\xi}, \bar{p}, \sim \bar{p}))^{\mathbf{4}} \in$ IPC $^{+}$ and $(\psi(\bar{\xi}, \sim \bar{\xi}, \bar{p}, \sim \bar{p}))^{\mathbf{4}} \notin \mathbf{I P C} \mathbf{C}^{+}$. Taking into account that the translation $(\cdot)^{4}$ commutes with positive connectives we obtain $\varphi\left((\bar{\xi})^{\mathbf{4}},(\sim \bar{\xi})^{\mathbf{4}}, \bar{p}, \bar{p}^{d}\right) \in$ IPC $^{+}$and $\psi\left((\bar{\xi})^{\mathbf{4}},(\sim \bar{\xi})^{\mathbf{4}}, \bar{p}, \bar{p}^{d}\right) \notin$ IPC $^{+}$. By induction on the structure of formulas we can easily check that $(\sim \theta)^{\mathbf{4}}=\left(\theta^{\mathbf{4}}\right)^{d}$, which allows us to conclude that

$$
\varphi\left(\bar{\xi}^{\mathbf{4}},\left(\bar{\xi}^{\mathbf{4}}\right)^{d}, \bar{p}, \bar{p}^{d}\right) \in \mathbf{I P C} \mathbf{C}^{+} \text {and } \psi\left(\bar{\xi}^{\mathbf{4}},\left(\bar{\xi}^{\mathbf{4}}\right)^{d}, \bar{p}, \bar{p}^{d}\right) \notin \mathbf{I P C} \mathbf{C}^{+}
$$

We have thus proved that $d$-admissibility in $\mathbf{I P C}^{+}$implies admissibility in N4.

To prove the inverse implication assume that $\varphi\left(\bar{\xi}, \bar{\xi}^{d}, \bar{p}, \bar{p}^{d}\right) \in \mathbf{I P C}^{+}$and $\psi\left(\bar{\xi}, \bar{\xi} \bar{\xi}^{d}, \bar{p}, \bar{p}^{d}\right) \notin$ IPC $^{+}$for a tuple $\bar{\xi}$ of positive formulas.

For a formula $\theta \in \mathrm{Fm}^{+}\left(\operatorname{Prop} \cup \operatorname{Prop}^{\dagger}\right)$, we denote by $\theta^{\prime}$ the result of replacement of every variable $p_{i}^{\dagger}$ by $\sim p_{i}$. It is clear that $\theta^{\prime} \in F m^{\sim}$ and $\left(\theta^{\prime}\right)^{4}=\theta$. Using this fact we can prove by induction on the structure of formulas that for every $\theta \in F m^{+}\left(\operatorname{Prop} \cup \operatorname{Prop}^{\dagger}\right)$, we have $\left(\sim \theta^{\prime}\right)^{\mathbf{4}}=\theta^{d}$. From this fact and the definition of translation $(\cdot)^{\mathbf{4}}$ we obtain

$$
\left(\varphi\left(\bar{\xi}^{\prime}, \sim \bar{\xi}^{\prime}, \bar{p}, \sim \bar{p}\right)\right)^{4}=\varphi\left(\bar{\xi}, \bar{\xi}^{d}, \bar{p}, \bar{p}^{d}\right) .
$$

Consequently, $\varphi\left(\bar{\xi}^{\prime}, \sim \bar{\xi}^{\prime}, \bar{p}, \sim \bar{p}\right) \in \mathbf{N} 4$. In a similar way, $\psi\left(\bar{\xi}^{\prime}, \sim \bar{\xi}^{\prime}, \bar{p}, \sim \bar{p}\right) \notin$ N4. We have thus proved that the rule $\frac{\varphi(\bar{x}, \sim \bar{x}, \bar{p}, \sim \bar{p})}{\psi(\bar{x}, \sim \bar{x}, \bar{p}, \sim \bar{p})}$ is not admissible in N4.

The admissibility problem for the logics $\mathbf{N} 4$ and $\mathbf{N} \mathbf{4}^{\perp}$ is reduced in this way to $d$-admissibilty problem for positive and intuitionistic logics. We are not ready to solve either of these problems, but in view of the fact that literals $p_{i}$ and $\sim p_{i}$ are interpreted independently in $\mathbf{N} \mathbf{4}$-models there is a sense to consider a weak form of admissibility, which assumes that literals $p$ and $\sim p$ are replaced not by formulas $\xi$ and $\sim \xi$, but by arbitrary formulas $\xi$ and $\zeta$. We say that an inference rule

$$
\frac{\varphi(\bar{x}, \sim \bar{x}, \bar{p}, \sim \bar{p})}{\psi(\bar{x}, \sim \bar{x}, \bar{p}, \sim \bar{p})}
$$


is weakly admissible in $\mathbf{N 4}\left(\mathbf{N} 4^{\perp}\right)$ if for every tuples of formulas $\bar{\xi}$ and $\bar{\zeta}$ of the same length as $\bar{x}$, we have $\psi(\bar{\xi}, \bar{\zeta}, \bar{p}, \sim \bar{p}) \in \mathbf{N} 4\left(\mathbf{N} 4^{\perp}\right)$, whenever $\varphi(\bar{\xi}, \bar{\zeta}, \bar{p}, \sim \bar{p}) \in \mathbf{N} 4\left(\mathbf{N} 4^{\perp}\right)$.

The weak admissibility problem for $\mathbf{N} 4\left(\mathbf{N} 4^{\perp}\right)$ is equivalent to admissibility problem for positive intuitionistic logic (intuitionistic logic).

Proposition 4.2. Let $\varphi(\bar{x}, \bar{y}, \bar{p}, \bar{q}), \psi(\bar{x}, \bar{y}, \bar{p}, \bar{q}) \in F m^{+}(\operatorname{Prop})\left(F m^{\perp}(\operatorname{Prop})\right)$. The rule

$$
\frac{\varphi(\bar{x}, \sim \bar{x}, \bar{p}, \sim \bar{p})}{\psi(\bar{x}, \sim \bar{x}, \bar{p}, \sim \bar{p})}
$$

is weakly admissible in $\mathbf{N} 4\left(\mathbf{N} \mathbf{4}^{\perp}\right)$ iff the rule

$$
\frac{\varphi\left(\bar{x}, \bar{y}, \bar{p}, \bar{p}^{d}\right)}{\psi\left(\bar{x}, \bar{y}, \bar{p}, \bar{p}^{d}\right)}
$$

is admissible in $\mathbf{I P C} \mathbf{C}^{+}$(IPC).

Proof. Again, we will consider only the case of $\mathbf{N} 4$ and $\mathbf{I P C} \mathbf{C}^{+}$.

Assume that tuples of formulas $\bar{\xi}$ and $\bar{\zeta}$ are such that $\varphi(\bar{\xi}, \bar{\zeta}, \bar{p}, \sim \bar{p}) \in$ $\mathbf{N 4}$, but $\psi(\bar{\xi}, \bar{\zeta}, \bar{p}, \sim \bar{p}) \notin \mathbf{N 4}$. By Theorem 2.1 we have $(\varphi(\bar{\xi}, \bar{\zeta}, \bar{p}, \sim \bar{p}))^{\mathbf{4}} \in$ IPC $^{+}$and $(\psi(\bar{\xi}, \bar{\zeta}, \bar{p}, \sim \bar{p}))^{\mathbf{4}} \notin$ IPC $^{+}$. Since $(\cdot)^{\mathbf{4}}$ commutes with positive connectives and send $p_{i}$ to $p_{i}^{\dagger}$, we have $\varphi\left((\bar{\xi})^{\mathbf{4}},(\bar{\zeta})^{\mathbf{4}}, \bar{p}, \bar{p}^{d}\right) \in \mathbf{I P C}^{+}$and $\psi\left((\bar{\xi})^{\mathbf{4}},(\bar{\zeta})^{\mathbf{4}}, \bar{p}, \bar{p}^{d}\right) \notin \mathbf{I P C}^{+}$. The latter means that admissibility in $\mathbf{I P C}^{+}$ implies weak admissibility in $\mathbf{N} 4$.

Now we assume that $\varphi\left(\bar{\xi}, \bar{\zeta}, \bar{p}, \bar{p}^{d}\right) \in \mathbf{I P C} \mathbf{C}^{+}$and $\psi\left(\bar{\xi}, \bar{\zeta}, \bar{p}, \bar{p}^{d}\right) \notin \mathbf{I P C} \mathbf{C}^{+}$ for tuples $\bar{\xi}$ and $\bar{\zeta}$ of positive formulas with variables from Prop $\cup$ Prop ${ }^{\dagger}$. The formula $\theta^{\prime}$ is defined as in the proof of the previous proposition. Recall that $\left(\theta^{\prime}\right)^{\mathbf{4}}=\theta$. By definition of $(\cdot)^{4}$ we have

$$
\left(\varphi\left(\bar{\xi}^{\prime}, \bar{\zeta}^{\prime}, \bar{p}, \sim \bar{p}\right)\right)^{\mathbf{4}}=\varphi\left(\bar{\xi}, \bar{\zeta}, \bar{p}, \bar{p}^{d}\right) \text { and }\left(\psi\left(\bar{\xi}^{\prime}, \bar{\zeta}^{\prime}, \bar{p}, \sim \bar{p}\right)\right)^{\mathbf{4}}=\psi\left(\bar{\xi}, \bar{\zeta}, \bar{p}, \bar{p}^{d}\right),
$$

whence $\varphi\left(\bar{\xi}^{\prime}, \bar{\zeta}^{\prime}, \bar{p}, \sim \bar{p}\right) \in \mathbf{N} 4$ and $\psi\left(\bar{\xi}^{\prime}, \bar{\zeta}^{\prime}, \bar{p}, \sim \bar{p}\right) \notin \mathbf{N 4}$, which proves that the rule $\frac{\varphi(\bar{x}, \sim \bar{x}, \bar{p}, \sim \bar{p})}{\psi(\bar{x}, \sim \bar{x}, \bar{p}, \sim \bar{p})}$ is not weakly admissible in $\mathbf{N} 4$.

From this proposition and the facts that the problems of admissibility of rules with parameters for positive logic $\mathbf{I P C}^{+}[24]$ and for intuitionistic logic IPC [31] are algorithmically decidable we obtain the following

Corollary 4.3. The problems of weak admissibility of rules with parameters for Nelson's logics $\mathbf{N} 4$ and $\mathbf{N} \mathbf{4}^{\perp}$ are algorithmically decidable.

\section{Weak admissibility of rules in $\mathrm{N} 3, \mathrm{~N} 4^{\mathrm{N}}$, and $\mathrm{N} 4^{\circ}$}

Earlier we have defined the notion of weak admissibility specially for the logics $\mathbf{N} 4$ and $\mathbf{N} 4^{\perp}$, because this notion reflects a unique semantic feature of these logics: the interpretations of literals $V(p)$ and $V(\sim p)$ are completely independent one from the other. Passing from $\mathbf{N} 4$ and $\mathbf{N} 4^{\perp}$ to their extensions will impose restrictions on the interrelations between $V(p)$ and $V(\sim p)$, so 
the adequate notion of weak admissibility for these logics must reflect these restrictions. In this section we define and investigate the notion of weak admissibility for the logics $\mathbf{N} 3, \mathbf{N} 4^{\mathrm{N}}$, and $\mathbf{N} 4^{\circ}$. The class of N3-models is distinguished in the class of all $\mathbf{N 4}$-models by the requirement $V(p) \cap V(\sim p)=\varnothing$, which reflects the fact that $\neg(p \wedge \sim p) \in \mathbf{N 3}$. Recall that here the symbol $\neg$ denotes the intuitionistic negation definable in N3 as $\neg \varphi:=\varphi \rightarrow \perp$. This restriction has the following natural syntactical correspondence: instead of $p$ and $\sim p$ we have to substitute a pair of inconsistent formulas $\xi$ and $\zeta$, i.e. $\neg(\xi \wedge \zeta) \in \mathbf{N} 3$. These considerations lead to the following definition.

Let $\varphi(\bar{x}, \bar{y}, \bar{p}, \bar{q}), \psi(\bar{x}, \bar{y}, \bar{p}, \bar{q}) \in F m^{\perp}($ Prop $)$. We say that the inference rule

$$
\frac{\varphi(\bar{x}, \sim \bar{x}, \bar{p}, \sim \bar{p})}{\psi(\bar{x}, \sim \bar{x}, \bar{p}, \sim \bar{p})}
$$

with parameters is weakly admissible in N3 if for every tuples of formulas $\bar{\xi}$ and $\bar{\zeta}$ of the same length as $\bar{x}$ and such that $\neg(\bar{\xi} \wedge \bar{\zeta}) \in \mathbf{N 3}$, we have $\psi(\bar{\xi}, \bar{\zeta}, \bar{p}, \sim \bar{p}) \in \mathbf{N} 3$, whenever $\varphi(\bar{\xi}, \bar{\zeta}, \bar{p}, \sim \bar{p}) \in \mathbf{N} 3$. Naturally, the denotation $\neg(\bar{\xi} \wedge \bar{\zeta}) \in \mathbf{N} 3$ means that $\neg\left(\xi_{i} \wedge \zeta_{i}\right) \in \mathbf{N 3}$ for all respective components $\xi_{i}$ and $\zeta_{i}$ of tuples $\bar{\xi}$ and $\bar{\zeta}$.

The class of $\mathbf{N} 4^{\mathrm{N}}$-models is distinguished in the class of all $\mathbf{N} 4$-models by the requirement that $(V(p) \cup V(\sim p)) \downarrow$ coincides with the set of all worlds, which corresponds to $\neg \neg(p \vee \sim p) \in \mathbf{N} 4^{\mathrm{N}}$. In case of finite models this requirement can be replaced by the following: all maximal worlds are in $V(p) \cup V(\sim p)$. The class of $\mathbf{N} 4^{\circ}$-models is the intersection of classes of N3- and $\mathbf{N} 4^{\mathrm{N}}$-models. Naturally, we define the weak admissibility of rules for these logics as follows.

We say that the inference rule

$$
\frac{\varphi(\bar{x}, \sim \bar{x}, \bar{p}, \sim \bar{p})}{\psi(\bar{x}, \sim \bar{x}, \bar{p}, \sim \bar{p})}
$$

with parameters is weakly admissible in $\mathbf{N} 4^{\mathrm{N}}\left(\mathbf{N} 4^{\circ}\right)$ if for every tuples of formulas $\bar{\xi}$ and $\bar{\zeta}$ of the same length as $\bar{x}$ and such that $\neg \neg(\bar{\xi} \vee \bar{\zeta}) \in \mathbf{N} 4^{\mathrm{N}}$ $\left(\neg \neg(\bar{\xi} \vee \bar{\zeta}) \in \mathbf{N} 4^{\circ}\right.$ and $\left.\neg(\bar{\xi} \wedge \bar{\zeta}) \in \mathbf{N} 4^{\circ}\right)$, we have $\psi(\bar{\xi}, \bar{\zeta}, \bar{p}, \sim \bar{p}) \in \mathbf{N 3}$, whenever $\varphi(\bar{\xi}, \bar{\zeta}, \bar{p}, \sim \bar{p}) \in \mathbf{N} 3$.

Unfortunately, we do not see a possibility to reduce the weak admissibility problem for $\mathbf{N} 3, \mathbf{N} 4^{\mathrm{N}}$, and $\mathbf{N} 4^{\circ}$ to the solved admissibility problem for intuitionistic logic in a way similar to that for paraconsistent versions of Nelson logic. Due to this reason we adapt a technique from [24].

For a rule $r=\varphi(\bar{x}, \sim \bar{x}, \bar{p}, \sim \bar{p}) / \psi(\bar{x}, \sim \bar{x}, \bar{p}, \sim \bar{p})$, denote by $S u b(r)$ the set $\operatorname{Sub}(\{\varphi, \psi\})$, by $V P(r)$ the set of all variables and parameters and by $P(r)$ the set of all parameters of the rule $r$. We put $\operatorname{Lit}(r)=V P(r) \cup \sim V P(r)$ and $\operatorname{Lit} P(r)=P(r) \cup \sim P(r)$.

For every subset $M \subseteq 2^{S u b(r)}$, we define an N4-model $\mathcal{M}=\langle M, \subseteq, V\rangle$, where the valuation $V: \operatorname{Lit}(r) \rightarrow 2^{M}$ is defined so that $V(\alpha)=\{X \in$ $M \mid \alpha \in X\}$ for $\alpha \in \operatorname{Lit}(r)$. It is clear that $\mathcal{M}$ is an $\mathbf{N 3 - m o d e l ~ i f f ~ a l l ~}$ $X \in M$ are consistent w.r.t. literals, i.e. there are no $p_{i}$ and $X \in M$ such 
that $\left\{p_{i}, \sim p_{i}\right\} \subseteq X . \mathcal{M}$ is an $\mathbf{N} 4^{\mathbf{N}}$-model iff for every maximal $X \in M$ end every $p_{i} \in V P(r)$ either $p_{i}$ or $\sim p_{i}$ is in $X$.

Recall that (just by definition) if an inference rule $r=\varphi(\bar{x}, \sim \bar{x}, \bar{p}, \sim$ $\bar{p}) / \psi(\bar{x}, \sim \bar{x}, \bar{p}, \sim \bar{p})$ is not admissible in $\mathbf{N 3}\left(\mathbf{N} 4^{\mathrm{N}}, \mathbf{N} 4^{\circ}\right)$, then there is a model $\mathcal{N}^{\mathbf{3}}(n)\left(\mathcal{N}^{\mathrm{N}}(n), \mathcal{N}^{\circ}(n)\right)$, where $P_{n}$ contains all $P(r)$, and a definable N3- $\left(\mathbf{N} 4^{\mathrm{N}}-, \mathbf{N} 4^{\circ}\right.$-) valuation $V_{1}$ coinciding with the original valuation of $\mathcal{N}^{3}(n)\left(\mathcal{N}^{\mathrm{N}}(n), \mathcal{N}^{\circ}(n)\right)$ on literals from $\operatorname{Lit} P(r)$, where $\varphi$ is true w.r.t. $V_{1}$ at all worlds from $\mathcal{N}(n)$, but $\psi$ is false w.r.t. $V_{1}$ at some world.

Proposition 5.1. Let $r=\varphi(\bar{x}, \sim \bar{x}, \bar{p}, \sim \bar{p}) / \psi(\bar{x}, \sim \bar{x}, \bar{p}, \sim \bar{p})$ be an inference rule with parameters and let $n$ be such that $P(r) \subset P_{n}$. Suppose that, for an arbitrary $\mathbf{N} 3-\left(\mathbf{N} 4^{\mathrm{N}}-, \mathbf{N}^{\circ}{ }^{-}\right.$) valuation $V_{1}$ of all literals from $r$ in $\mathcal{N}^{\mathbf{3}}(n)$ $\left(\mathcal{N}^{\mathrm{N}}(n), \mathcal{N}^{\circ}(n)\right)$ which coincides with the original valuation of $\mathcal{N}^{\mathbf{3}}(n)\left(\mathcal{N}^{\mathrm{N}}(n)\right.$, $\left.\mathcal{N}^{\circ}(n)\right)$ on $\operatorname{LitP}(r), V_{1}(\varphi)=N^{\mathbf{3}}(n)\left(N^{\mathrm{N}}(n), N^{\circ}(n)\right)$ and $V_{1}(\psi) \neq N^{\mathbf{3}}(n)$ $\left(N^{\mathrm{N}}(n), N^{\circ}(n)\right)$. Then there exists $M \subseteq 2^{S u b(r)}$ such that the $\mathbf{N} 3-\left(\mathbf{N}^{\mathrm{N}}-\right.$, $\mathbf{N} 4^{\circ}{ }^{-}$) model $\mathcal{M}=\langle M, \subseteq, V\rangle$ has the following properties:

(a) $\forall \xi \in S u b(r) \forall X \in M(\mathcal{M}, X \Vdash \xi \Leftrightarrow \xi \in X)$

(b) $\forall \nabla \subseteq M \forall b \subseteq \bigcap\left\{\operatorname{Lit}_{\mathcal{M}}(X) \cap \operatorname{Lit} P(r) \mid X \in \nabla\right\} \quad$ (or $\forall b \subseteq \operatorname{Lit} P(r)$ if $\nabla$ is empty) if $b$ is consistent, then

$$
\exists Y \in M\left(b=\operatorname{Lit}_{\mathcal{M}}(Y) \cap \operatorname{LitP}(r) \&\right.
$$

$[\nabla=\varnothing \Rightarrow(\xi \rightarrow \zeta \in S u b(r) \Rightarrow(\xi \rightarrow \zeta \in Y \Leftrightarrow(\xi \notin Y \vee \zeta \in Y)))]$

$[\nabla \neq \varnothing \Rightarrow Y \subseteq \bigcap\{X \mid X \in \nabla\}] \&((\xi \rightarrow \zeta \in S u b(r) \& \xi \rightarrow \zeta \notin Y) \Rightarrow$ $((\xi \in Y \& \zeta \notin Y) \vee \exists X \in \nabla(\xi \rightarrow \zeta \notin X)))$;

(c) $\forall X \in M(\varphi \in X) \& \psi \notin X_{m}$, where $X_{m}:=\inf \langle M, \subseteq\rangle$.

Proof. Let us consider an arbitrary N3-valuation $V_{1}$ in $\mathcal{N}^{\mathbf{3}}(n)$ for all letters from $r$, which agrees with the original valuation $V^{(n)}$ on $\operatorname{Lit} P(r)$, where $V_{1}(\varphi)=N(n)$ and $V_{1}(\psi) \neq N(n)$.

For every $a \in N^{\mathbf{3}}(n)$, we put

$$
t(a):=\left\{\xi \in S u b(r) \mid a \Vdash_{V_{1}} \xi\right\} .
$$

The set $M$ is defined as $\left\{t(a) \mid a \in N^{\mathbf{3}}(n)\right\}$. Since $V_{1}$ is an N3-valuation, the respective $\mathcal{M}$ is an $\mathbf{N} 3$-model. We prove that $\mathcal{M}$ satisfies all the required properties.

We, first, proof the property (a) by induction on the length of the formula $\xi$. For elements of $\operatorname{Lit}(r)$ the desired equivalence holds by definition of the valuation $V$ in the model $\mathcal{M}$. Consider the case of complex formulas. Let $\mathcal{M}, X \Vdash \xi \wedge \zeta$. Then $\mathcal{M}, X \Vdash \xi$ and $\mathcal{M}, X \Vdash \zeta$, and we have by induction hypothesis $\xi \in X$ and $\zeta \in X$. By definition of $\mathcal{M}$ there is $a \in N^{\mathbf{3}}(n)$ such that $X=t(a)$, and we have $a \Vdash_{V_{1}} \xi$ and $a \Vdash_{V_{1}} \zeta$. Consequently, $a \Vdash_{V_{1}} \xi \wedge \zeta$, i.e., $\xi \wedge \zeta \in X$. On the other hand, if $\xi \wedge \zeta \in X$ and $X=t(a)$, then $a \Vdash_{V_{1}} \xi$ and $a \Vdash_{V_{1}} \zeta$. Therefore, $\xi \in X$ and $\zeta \in X$. By the induction hypothesis we have $\mathcal{M}, X \Vdash \xi$ and $\mathcal{M}, X \Vdash \zeta$, which implies $\mathcal{M}, X \Vdash \xi \wedge \zeta$.

The case of disjunction can be treated in a similar way.

Since $\varphi$ and $\psi$ are $n n f$, we do not need to consider the cases of negated formulas. So it remains only to consider the case of implication. Let $\xi \rightarrow \zeta \in$ 
$X$. If $X \subseteq Y$ and $\mathcal{M}, Y \Vdash \xi$, then $\xi \rightarrow \zeta \in Y$ and $\xi \in Y$ by the induction hypothesis. Since $Y=t(a)$ for some $a$, we have $\zeta \in Y$ and hence $\mathcal{M}, Y \Vdash \zeta$. Thus, $\mathcal{M}, X \Vdash \xi \rightarrow \zeta$. Conversely, suppose that $\xi \rightarrow \zeta \notin X$. Since $X=t(a)$ for some $a$, we have $a \nVdash_{V_{1}} \xi \rightarrow \zeta$. Consequently, there is an element $b$ such that $a \leq b, b \Vdash_{V_{1}} \xi$ and $b \nVdash_{V_{1}} \zeta$. By induction hypothesis we have $\mathcal{M}, t(b) \Vdash \xi$ and $\mathcal{M}, t(b) \nVdash \zeta$. Moreover, $X=t(a) \subseteq t(b)$, consequently, $\mathcal{M}, X \nVdash \xi \rightarrow \zeta$. The property (a) is thus proved.

We proceed to the proof of the property (b). Assume that $\nabla=\varnothing$. By construction of the $n$-characterizing model $\mathcal{N}^{\mathbf{3}}(n)$, for every consistent $b \subseteq \operatorname{Lit} P(r)$, there is a maximal in $\mathcal{N}^{\mathbf{3}}(n)$ element $a$ such that $\operatorname{Lit}_{\mathcal{N}(n)}(a)=b$ (since $\left.P(r) \subset P_{n}\right)$. Since $V^{(n)}$ and $V_{1}$ agree on $\operatorname{Lit} P(r), \operatorname{Lit}_{\mathcal{M}}(t(a))=b$. Finally, the maximality of $a$ implies that $\xi \rightarrow \zeta \in t(a)$ iff $\xi \notin t(a)$ or $\zeta \in t(a))$.

Consider the case $\nabla \neq \varnothing$. Let $\nabla=\left\{t\left(a_{j}\right) \mid a_{j} \in N^{\mathbf{3}}(n), j \in J\right\}$, and

$$
b \subseteq \bigcap\left\{\operatorname{Lit}_{\mathcal{M}}(X) \cap \operatorname{Lit} P(r) \mid X \in \nabla\right\} .
$$

By the construction of the model $\mathcal{N}^{\mathbf{3}}(n)$, there exists $c \in N^{\mathbf{3}}(n)$ such that $[c]=\{c\} \cup \bigcup\left\{\left[a_{j}\right] \mid j \in J\right\}$. Moreover, if $b \varsubsetneqq \bigcap \operatorname{Lit}_{\mathcal{M}}\left(t\left(a_{j}\right)\right) \cap \operatorname{LitP}(r) \mid$ $j \in J\}$ or if there is no smallest element among the $a_{j}$ 's w.r.t. $\leq$, then there exists $c$ with the property $\operatorname{Lit}_{\mathcal{M}}(c)=b$. In the other case, when a smallest element does exists and the above property for $b$ is not true, we take this smallest element as $c$. We define $Y:=t(c)$. The truth of all the necessary properties can be verified directly.

By the condition of the theorem for all $a \in N^{\mathbf{3}}(n)$, we have $a \Vdash_{V_{1}} \varphi$, i.e., $\varphi \in t(a)$ for all $a$. On the other hand, there exists $b \in N^{3}(n)$ such that $b \nVdash_{V_{1}} \psi$. So, $\psi \notin t(b)$. By (b) there exists a smallest element $X_{m}$ in $\mathrm{M}$, and $X_{m} \subseteq t(b)$ implies $\psi \notin X_{m}$. So, property (c) is also true.

It is clear that if $V_{1}$ is an $\mathbf{N} 4^{\mathrm{N}}-\left(\mathbf{N} 4^{\circ}\right.$-) valuation in $\mathcal{N}^{\mathbf{3}}(n)$, then the model $\mathcal{M}$ defined as above is an $\mathbf{N} 4^{\mathrm{N}}-\left(\mathbf{N} 4^{\circ}-\right)$ model. The rest of the proof is the same.

AAAAAA The same as what?

The next theorem plays the central part for deciding admissibility.

Theorem 5.2. If $r=\varphi(\bar{x}, \sim \bar{x}, \bar{p}, \sim \bar{p}) / \psi(\bar{x}, \sim \bar{x}, \bar{p}, \sim \bar{p})$ is an inference rule with parameters and there exists $M \subseteq 2^{\text {Sub(r) }}$ such that $\mathcal{M}=\langle M, \subseteq, V\rangle$ is an N3-(N4 ${ }^{\mathrm{N}}$-, $\mathbf{N 4}^{\circ}$-) model and has properties (a)-(c) from Proposition 5.1 , then for some $n$, where $P(r) \subset P_{n}$, there exist formulas $\bar{\xi}$ and $\bar{\zeta}$ in propositional letters from $P_{n}$ such that $\neg(\bar{\xi} \wedge \bar{\zeta}) \in \mathbf{N} 3\left(\neg(\bar{\xi} \wedge \bar{\zeta}) \in \mathbf{N} 4^{\mathbf{N}}\right.$, respectively $\neg(\bar{\xi} \wedge$ $\bar{\zeta}) \in \mathbf{N} 4^{\circ}$ and $\left.\neg(\bar{\xi} \wedge \bar{\zeta}) \in \mathbf{N} 4^{\circ}\right)$ and

$$
\varphi(\bar{\xi}, \bar{\zeta}, \bar{p}, \sim \bar{p}) \in \mathbf{N} 3\left(\mathbf{N} 4^{\mathrm{N}}, \mathbf{N} 4^{\circ}\right), \quad \psi(\bar{\xi}, \bar{\zeta}, \bar{p}, \sim \bar{p}) \notin \mathbf{N} 3\left(\mathbf{N} 4^{\mathbf{N}}, \mathbf{N} 4^{\circ}\right) .
$$

Moreover, formulas $\bar{\xi}$ and $\bar{\zeta}$ can be constructed effectively in $\mathcal{M}$.

Proof. First we consider the logic N3. Let $M \subseteq 2^{S u b(r)}$ be such that $\mathcal{M}=$ $\langle M, \subseteq, V\rangle$ is an N3-model and has properties (a)-(c) from Proposition 5.1. 
Take the model $\mathcal{N}^{\mathbf{3}}(n)$, where $n=\|M\|+\|$ LitP $(r) \|$. We assume any $X$ from $\mathrm{M}$ to be associated with the letter $p_{X}$ from $\operatorname{Dom}\left(V^{(n)}\right)$, and $P(r)$ just to be letters from $\operatorname{Dom}\left(V^{(n)}\right)$ of $\mathcal{N}^{\mathbf{3}}(n)$. Let us extend the valuation $V$ of the model $\mathcal{M}$ to all variables $p_{X}, X \in M$, by putting $V\left(p_{X}\right):=\{Y \in M \mid Y \nsubseteq X\}$ and $V\left(\sim p_{X}\right):=\varnothing$. Since $V\left(\sim p_{X}\right):=\varnothing$ it will be again an N3-valuation. In what follows $\mathcal{M}$ denotes a model with the extended valuation, in particular, atoms of the form $p_{X}$ are true or false at worlds of $\mathcal{M}$ and a literals $\sim p_{X}$ are false at worlds of $\mathcal{M}$.

Our goal is to construct a definable N3-valuation $V_{1}$ of $\mathcal{N}^{\mathbf{3}}(n)$ such that $V_{1}$ agrees with $V^{(n)}$ on $\operatorname{Lit} P(r)$ and satisfies:

$$
V_{1}(\varphi(\bar{x}, \sim \bar{x}, \bar{p}, \sim \bar{p}))=N^{\mathbf{3}}(n) \text { and } V_{1}\left(\psi(\varphi(\bar{x}, \sim \bar{x}, \bar{p}, \sim \bar{p})) \neq N^{\mathbf{3}}(n) .\right.
$$

To this end we construct an ascending sequence of N3-models $e_{k}(\mathcal{M}), k \in \omega$ (which are based at cones of $\mathcal{N}^{\mathbf{3}}(n)$ ) with definable in $\mathcal{N}^{\mathbf{3}}(n)$ valuations, which agree with $V^{(n)}$ on $\operatorname{Lit} P(r)$. Moreover, for every $k \in \omega$, it will be $e_{k}(\mathcal{M})=\bigcup_{Y \in M} e_{k}(Y)$, where any $e_{k}(Y)$ is a definable cone of $\mathcal{N}^{\mathbf{3}}(n)$. Finally, we will need that for every $k \geq 0$ the following conditions are satisfied.

(p1) $e_{k-1}(X) \subseteq e_{k}(X)$ for all $X \in M$. Here we assume $e_{-1}(X)=\varnothing$ and $e_{-1}(\mathcal{M})=\varnothing$.

(p2) For all $a \in e_{k}(\mathcal{M}) \backslash e_{k-1}(\mathcal{M})$ there is a unique $X(a, k)$ in $\mathcal{M}$ such that: $a \in e_{k}(X(a, k)) \backslash e_{k-1}(\mathcal{M})$ and $X(a, k+1)$ is greatest among all $Y$, where $a \in e_{k}(Y) \backslash e_{k-1}(\mathcal{M})$.

(p3) If $c \in \mathcal{N}^{\mathbf{3}}(n), c \notin e_{k}(\mathcal{M})$, and

$$
\begin{aligned}
& M^{k}(c):=\left\{Y \in M \mid \exists a \exists j \in[0, k] c<a, a \in e_{j}(X(a, j)) \backslash e_{j-1}(\mathcal{M})\right\}, \\
& \text { then }\left\|M^{k}(c)\right\| \geq k+1 .
\end{aligned}
$$

(p4) The valuation $V_{k}$ of $e_{k}(\mathcal{M})$ is defined on $\operatorname{Lit}(r)$. It agrees with $V^{(n)}$ on $\operatorname{LitP}(r)$, whereas for literals $\alpha$ of the form $z$ or $\sim z$, where $z$ is variable letter of $r$, we have

$$
V_{k}(\alpha):=\bigcup_{0 \leq j \leq k}\left\{e_{j}(Y) \mid \alpha \in Y, Y \in M\right\} ;
$$

$V_{k}$ coincides with any $V_{j}$ on $e_{j}(\mathcal{M})$ for $j<k$. In this way, $e_{j}(\mathcal{M})$ is a generated submodel of $e_{k}(\mathcal{M})$.

(p5) $\forall l \leq k, \forall \varphi \in S u b(r), \forall a \in e_{l}(\mathcal{M}) \backslash e_{l-1}(\mathcal{M})\left(e_{-1}(\mathcal{M})=\varnothing\right.$, cf. $(\mathrm{p} 1)$ above)

$$
\left[e_{l}(\mathcal{M}), a \Vdash_{V_{l}} \varphi \Leftrightarrow \varphi \in X(a, l)\right] .
$$

Let us consider the case $k=0$ and define the model $e_{0}(\mathcal{M})$. By choice of $n$ there is no world $X$ of $\mathcal{M}$ such that $(\mathcal{M}, X) \vDash_{V} \wedge$ Lit $_{n}$ (cf. the definition of $V$ for new literals $p_{X}$ and $\sim p_{X}, X \in M$, in $\left.\mathcal{M}\right)$. If $X, Y \in \mathcal{M}$ and the sets $X$ and $Y$ are incomparable w.r.t. inclusion, then $X \Vdash_{V} p_{Y}, X \nVdash_{V} p_{X}$, $Y \Vdash_{V} p_{X}$, and $Y \nVdash_{V} p_{Y}$, i.e., $\operatorname{Lit}_{\mathcal{M}}(X) \neq \operatorname{Lit}_{\mathcal{M}}(Y)$. This implies that the model $\mathcal{M}$ has no twins. If $X, Y \in \mathcal{M}$ and $X \varsubsetneqq Y$, then $X \nVdash_{V} p_{X}$ and $Y \Vdash_{V} p_{X}$. Consequently, $\mathcal{M}$ has no duplications. 
We know from the proof of Proposition 3.1 that every finite N3-model with the above listed properties can be embedded into $\mathcal{N}^{\mathbf{3}}(n)$ as a generated submodel. Let $e$ be an embedding of $\mathcal{M}$ into $\mathcal{N}^{\mathbf{3}}(n)$. In this case, $e(\mathcal{M})$ is a cone of $\mathcal{N}^{3}(n)$, besides, by (a) from Proposition 5.1,

$$
\forall Y \in M \forall \varphi \in S u b(r)\left[\mathcal{N}^{\mathbf{3}}(n), e(Y) \Vdash_{V^{(n)}} \varphi \Leftrightarrow \varphi \in Y\right] .
$$

Now we will extend the model $e(\mathcal{M})$ to a model $e_{0}(\mathcal{M})$ containing all worlds of the model $\mathcal{N}^{\mathbf{3}}(n)$ with depth 1 . Note that since $\mathcal{M}$ is a finite object and $\mathcal{N}^{\mathbf{3}}(n)$ is effectively constructed, the construction of $e(\mathcal{M})$ is also effective, and by $c \in S^{1}\left(\mathcal{N}^{\mathbf{3}}(n)\right)$ we can effectively decide, whether $c \in e(\mathcal{M})$.

Take any $c \in S_{1}\left(\mathcal{N}^{\mathbf{3}}(n)\right) \backslash e(\mathcal{M})$, if one exists. By (b) from Proposition 5.1 there is $Y \in \mathcal{M}$ for $\nabla=\varnothing$ and $b:=\operatorname{Lit} P(r) \cap \operatorname{Lit}_{\mathcal{N}^{3}(n)}(c)$ such that $\operatorname{Lit} P(r) \cap \operatorname{Lit}_{\mathcal{M}}(Y)=\operatorname{LitP}(r) \cap \operatorname{Lit}_{\mathcal{N}^{\mathbf{3}}(n)}(c)$. We can effectively find one of such elements. Denote it by $X_{0}(c, 0)$. For every $Y \in M$, we put

$a_{0}(Y)=\left\{c \in S_{1}\left(\mathcal{N}^{\mathbf{3}}(n)\right) \backslash e(\mathcal{M}) \mid X_{0}(c, 0)=Y\right\}$ and $e_{0}(Y):=[e(Y)] \cup a_{0}(Y)$.

Since $X_{0}(c, 0)$ can be effectively found by $c$, we can effectively compute $e_{0}(Y)$ by $Y \in M$. Let $\bigcup_{Y \in M} e_{0}(Y)$ be the set of worlds of $e_{0}(\mathcal{M})$. We introduce the following valuation $V_{0}$ in $e_{0}(\mathcal{M})$ defined on $\operatorname{Lit}(r)$ : it agrees with $V^{(n)}$ on $\operatorname{LitP}(r)$, whereas for $\alpha=x_{j}$ or $\alpha=\sim x_{j}, x_{j} \in \bar{x}$, we put

$$
V_{0}(\alpha)=\left\{c \in S_{1}\left(\mathcal{N}^{\mathbf{3}}(n)\right) \backslash e(\mathcal{M}) \mid \mathcal{M}, X_{0}(c, 0) \Vdash \alpha\right\} \cup\left(e(\mathcal{M}) \cap V^{(n)}\left(x_{j}\right)\right) .
$$

Clearly, the cone $V_{0}(\alpha)$ can be effectively determined by $\alpha$. By Proposition 3.2 every sharp cone of $\mathcal{N}^{\mathbf{3}}(n)$ is $P_{n}$-definable, every $V_{0}(\alpha)$ is a finite union of sharp cones, therefore, it is also $P_{n}$-definable. We obtain that the valuation $V_{0}$ is definable in $\mathcal{N}^{\mathbf{3}}(n)$. Moreover, defining formulas for $V_{0}(\alpha)$ can be constructed effectively, because the cones $V_{0}(\alpha)$ are constructed effectively. For any $Y \in \mathcal{M}$, the cone $e_{0}(X)$ is definable in $\mathcal{N}^{\mathbf{3}}(n)$, and again we can effectively construct the formulas $s_{0}(Y)$ defining cones $e_{0}(Y)$. In every world of $e_{0}(\mathcal{M})$, the validity of literals is defined in the same way as in one of worlds of $\mathcal{M}$. Since $\mathcal{M}$ is an N3-model, $e_{0}(\mathcal{M})$ also is an N3-model.

We have thus constructed the $\mathbf{N 3}$-model $e_{0}(\mathcal{M})$ with a definable in $\mathcal{N}^{3}(n)$ valuation. Conditions (p1), (p3), and (p4) are satisfied for this model in an obvious way.

The sets $X_{0}(c, 0)$ were defined for all $c \in S_{1}\left(\mathcal{N}^{\mathbf{3}}(n)\right) \backslash e(\mathcal{M})$, and for such $c$, we set $X(c, 0):=X_{0}(c, 0)$. For $c \in e(\mathcal{M})$, we choose $X(c, 0)$ as a pre-image of $c$, w.r.t. $e$, i.e. $e\left(X_{0}(c, 0)\right)=c$. These sets $X(c, 0)$ validate condition $(\mathrm{p} 2)$.

Let $c \in S_{1}\left(\mathcal{N}^{\mathbf{3}}(n)\right) \backslash e(\mathcal{M})$. Since the set $X(c, 0)$ corresponds to the empty set $\nabla$, by item (b) of Proposition 5.1 we have $\xi \rightarrow \zeta \in X(c, 0)$ iff $\xi \notin X(c, 0)$ or $\zeta \in X(c, 0)$ for $\xi \rightarrow \zeta \in S u b(r)$. Since $c$ is a maximal element of $\mathcal{N}^{3}(n)$, we have $c \Vdash_{V_{0}} \xi \rightarrow \zeta$ iff $c \nVdash_{V_{0}} \xi$ or $c \Vdash_{V_{0}} \zeta$. This observation and definition of $V_{0}$ allow to prove that, for all such $c$,

$$
\forall \varphi \in S u b(r)\left[c \Vdash_{V_{0}} \varphi \Leftrightarrow \varphi \in X(c, 0)\right] .
$$

For $c \in e(\mathcal{M})$, the above equivalence holds in an obvious way. Thus, condition (p5) is verified. So, for $k=0$ all (p1) - (p5) hold. 


\section{Inductive step:}

Suppose that we constructed already for a given $k \geq 0$ and all $l \leq k$ the cones $e_{l}(Y)$ of $\mathcal{N}^{\mathbf{3}}(n)$ for $Y \in M$ such that any $e_{l}(Y)$ is definable in $\mathcal{N}^{\mathbf{3}}(n)$ by a formula $s_{l}(Y)$, and we defined the models $e_{l}(\mathcal{M}), l \leq k$, such that $\bigcup_{Y \in M} e_{l}(Y)$ is the set of worlds of $e_{l}(\mathcal{M})$ and conditions $(\mathrm{p} 1)-(\mathrm{p} 5)$ hold for $e_{l}(\mathcal{M})$.

Now we proceed to the construction of the model $e_{k+1}(\mathcal{M})$. Consider all $\nabla \subseteq M$, where $\|\nabla\|=k+1$. Let $\nabla=\left\{Y_{1}, \ldots, Y_{k+1}\right\}$.

Take an arbitrary subset $b$ of $\bigcap_{j \in[1, k+1]}\left(\operatorname{Lit}_{\mathcal{M}}\left(Y_{j}\right) \cap \operatorname{Lit} P(r)\right)$. For any pair $\nabla, b$ defined as above, there is $X_{\nabla, b} \in M$ satisfying property (b) of Proposition 5.1 for given $\nabla$ and $b$, fix it. Notice that if $\nabla$ contains the smallest set $Y$ and $b=b_{\nabla}=\bigcap_{j \in[1, k+1]}\left(Y_{j} \cap L i t P(r)\right)$ then we can choose as $X_{\nabla, b}$ this $Y$ and we do so (evidently this $Y$ satisfies property (b) of Proposition 5.1). We, in the sequel, will denote such $Y$ by $X_{\nabla, b_{\nabla}}^{\min }$. Otherwise, i.e. if $\nabla$ contains no smallest set or $b \neq \bigcap_{j \in[1, k+1]}\left(Y_{j} \cap \operatorname{Lit} P(r)\right)$, the set $X_{\nabla, b}$ does not belong to $\nabla$.

If $X_{\nabla, b_{\nabla}}^{\min }$ was defined for given $\nabla$, in particular, $X_{\nabla, b_{\nabla}}^{\min } \in \nabla$ we set:

$$
\begin{gathered}
\chi_{\min }^{\nabla}:=\bigwedge b_{\nabla} \wedge\left(\bigvee\left(\operatorname{Lit} P(r) \backslash b_{\nabla}\right) \rightarrow \bigvee_{Y \in M} s_{k}(Y)\right), \\
\psi^{\nabla}:=\bigwedge_{Y \notin \nabla} \bigwedge_{0 \leq j \leq k}\left[s_{j}(Y) \rightarrow \bigvee_{Y \subset Y_{1}} s_{j}\left(Y_{1}\right) \vee \bigvee_{Z \in M} s_{j-1}(Z)\right], \\
\varphi_{1}\left(\nabla, b_{\nabla}\right):=\chi_{m i n}^{\nabla} \wedge \psi^{\nabla}
\end{gathered}
$$

(for $s_{-1}(Z)$ we put $\perp$ ).

It is clear that $\chi_{\min }^{\nabla}$ is valid at the world $x$ of $\mathcal{N}^{\mathbf{3}}(n)$ iff all atoms from $b_{\nabla}$ are valid at $x$ and the validity of some atom from $\operatorname{Lit} P(r) \backslash b$ at $y \geq x$ implies $y \in e_{k}(\mathcal{M})$. The validity of $\psi^{\nabla}$ at $x$ means, in particular, that $M^{k}(x) \subseteq \nabla$.

If $X_{\nabla, b} \notin \nabla$, we define the formula $\psi^{\nabla}$ as above and put:

$$
\chi^{b, \nabla}:=\bigwedge b \wedge\left(\bigvee(\operatorname{Lit} P(r) \backslash b) \rightarrow \bigvee_{Y \in M} s_{k}(Y) \vee \phi(\nabla)\right),
$$

where $\phi(\nabla)=\varphi_{1}\left(\nabla, b_{\nabla}\right)$ if $\nabla$ contains the least element, otherwise $\phi(\nabla)=\perp$,

$$
\varphi_{2}(\nabla, b):=\chi^{b, \nabla} \wedge \psi^{\nabla}
$$

For any $Y \in M$ we set

$$
e_{k+1}(Y):=e_{k}(Y) \cup V^{(n)}\left(\bigvee_{Y=X_{\nabla, b}^{\min }} \varphi_{1}\left(\nabla, b_{\nabla}\right) \vee \bigvee_{Y=X_{\nabla, b}} \varphi_{2}(\nabla, b)\right)
$$

Lemma 5.3. Properties (p1) - (p5) hold for $e_{k+1}(Y), Y \in M$. 
Proof. It is clear that (p1) holds for $e_{k+1}(Y)$ for any $Y$.

For (p2):

let $a \in e_{k+1}(\mathcal{M}) \backslash e_{k}(\mathcal{M})$. We will need the following intermediate statement (it holds for any $k \geq 0$ ):

Proposition 5.4. If $a \in e_{k+1}(Y) \backslash e_{k}(\mathcal{M})$ then for $\nabla=M^{k}(a)$ either (i) $a \in e_{k+1}\left(X_{\nabla, b_{\nabla}}^{\min }\right) \backslash e_{k}(\mathcal{M})$ and $Y \subseteq X_{\nabla, b_{\nabla}}^{\min }$ or $(i i): a \notin e_{k+1}\left(X_{\nabla, b_{\nabla}}^{\min }\right) \backslash e_{k}(\mathcal{M})$ and $Y=X_{\nabla, b}$ for a unique $X_{\nabla, b}$, were $b \subseteq b_{\nabla}$.

Proof. If $a \in e_{k+1}(Y) \backslash e_{k}(\mathcal{M})$ then $a \Vdash_{V^{(n)}} \psi^{\nabla}$ for some $\nabla$. By our choice of $\psi^{\nabla}$ we have $M^{k}(a) \subseteq \nabla$. On the other hand by (p3) for $k$ there are at least $k+1$ different sets $Y_{j}$ in $M^{k}(a)$. Consequently, $M^{k}(a)=\nabla$. Thus, the family of sets $\nabla$ is uniquely determined by $a$. Then either (a) $a \Vdash_{V^{(n)}} \varphi_{1}\left(\nabla, b_{\nabla}\right)$ and $Y \subseteq X_{\nabla, b \nabla}^{\min }$ or (b) $a \Vdash_{V^{(n)}} \varphi_{2}(\nabla, b)$ and $Y=X_{\nabla, b}$ for some $X_{\nabla, b}$. If (a) does not hold, (b) holds and there is only one unique such $X_{\nabla, b}$.

In case (i) we set $X(a, k+1)=X_{\nabla, b \nabla}^{\min }$, in the case (ii) we put $X(a, k+1):=X_{\nabla, b}$. So, $(\mathrm{p} 2)$ is verified.

\section{For (p3):}

Let $c \in \mathcal{N}^{\mathbf{3}}(n) \backslash e_{k+1}(\mathcal{M})$. We have to prove $\left\|M^{k+1}(c)\right\| \geq k+2$. By (p3) for $k,\left\|M^{k}(c)\right\| \geq k+1$. Obviously, $M^{k}(c) \subseteq M^{k+1}(c)$, so $\left\|M^{k+1}(c)\right\| \geq k+1$. Assume $\left\|M^{k+1}(c)\right\|=k+1$, than $M^{k}(c)=M^{k+1}(c)$. Let $\nabla:=M^{k+1}(c)$, and for any element $a \in \mathcal{N}^{\mathbf{3}}(n), b_{a}:=\operatorname{Lit}_{\mathcal{N}^{\mathbf{3}}(n)}(a) \cap \operatorname{LitP}(r)$.

The equality $\nabla=M^{k}(c)$, in particular, means that for each $d>c$ such that $d \in e_{j}(Y) \backslash e_{j-1}(\mathcal{M})(j \leq k)$ for some $Y$, we have $d \in e_{j}(X(d, j)) \backslash e_{j}(\mathcal{M})$ for some $X(d, j) \in \nabla$. Therefore, we have

$$
c \Vdash_{V^{(n)}} \bigwedge_{Y \notin \nabla} \bigwedge_{0 \leq j \leq k}\left[s_{j}(Y) \rightarrow \bigvee_{Y \subset Y_{1}} s_{j}\left(Y_{1}\right) \vee \bigvee_{Z \in M} s_{j-1}(Z)\right]\left(=\psi^{\nabla}\right)
$$

Assume that $\nabla$ has no least element (smallest set) and consider a maximal $d \geq c$ with $d \notin e_{k}(\mathcal{M})$. Since $d \geq c$, we have $M^{k}(d) \subseteq M^{k}(c)=\nabla$.

At the same time $\left\|M^{k}(d)\right\| \geq k+1$ by $d \notin e_{k}(\mathcal{M})$ and (p3) for $k$, consequently, $M^{k}(d)=\nabla$. From the maximality of $d$, for $b_{d}=\operatorname{Lit}_{\mathcal{N}^{3}(n)}(d) \cap$ $\operatorname{Lit} P(r)$ we immediately obtain

$$
d \Vdash_{V^{(n)}} \bigwedge b_{d} \wedge\left(\bigvee\left(\operatorname{LitP}(r) \backslash b_{d}\right) \rightarrow \bigvee_{Y \in M} s_{k}(Y)\right)
$$

Since $\nabla$ has no least element, the last formula is equivalent to $\chi^{b_{d}, \nabla}$. We have also $d \Vdash \psi^{\nabla}$ by $c \leq d$. Consequently, $d \Vdash \varphi_{2}\left(\nabla, b_{d}\right)$ and $d \in e_{k+1}\left(X_{\nabla, b_{d}}\right)$. Moreover, since $\nabla$ has no least set, $X_{\nabla, b_{d}} \notin \nabla$. By Proposition 5.4 we have $X(d, k+1)=X_{\nabla, b_{d}}$ (since no $\left.X_{\nabla, b_{\nabla}}^{\min }\right)$. This contradicts the earlier proved equalities $M^{k+1}(c)=M^{k}(c)=\nabla$.

Consider now the case when $\nabla$ contains the smallest set. For any element $d$, where $c \leq d$ and $d \notin e_{k}(\mathcal{M})$ (in particular if $d \notin e_{k+1}(\mathcal{M})$ ), we may show 
$M^{k}(d)=\nabla=M^{k}(c)$ the same way as above. By $M^{k}(d)=\nabla$ and $X_{\nabla, b_{\nabla}}^{\min } \in \nabla$ we conclude $d \leq d_{1}$ for some $d_{1} \in e_{j}\left(X\left(d_{1}, j\right)\right) \backslash e_{j-1}(\mathcal{M})$ for $j \leq k$ and $X\left(d_{1}, j\right)=X_{\nabla, b_{\nabla}}^{\min }$. Consequently always $b_{d}=\operatorname{Lit}_{\mathcal{N}^{3}(n)}(d) \cap \operatorname{LitP}(r) \subseteq b_{\nabla}$. Therefore, if $b_{d}=b_{\nabla}$ we have

$$
d \Vdash_{V^{(n)}} \bigwedge b_{\nabla} \wedge\left(\bigvee\left(\operatorname{LitP}(r) \backslash b_{\nabla}\right) \rightarrow \bigvee_{Y \in M} s_{k}(Y)\right),
$$

and $d \in e_{k+1}\left(X_{\nabla, b_{\nabla}}^{\min }\right)$

In particular, it holds for all $d$, where $c \leq d, d \notin e_{k+1}(\mathcal{M})$ and $b_{d}=b_{\nabla}$. Thus if $b_{c}=b_{\nabla}$, we would have $c \in e_{k+1}\left(X_{\nabla, b_{\nabla}}^{\min }\right)$ - a contradiction, so $b_{c} \subset b_{\nabla}$.

Take any $c_{1}$ in $\langle c\rangle \backslash e_{k}(\mathcal{M})$, then as we noticed above $b_{c_{1}} \subseteq b_{\nabla}$, and if $b_{c_{1}}=b_{\nabla}$ we have $c_{1} \in e_{k+1}\left(X_{\nabla, b_{\nabla}}^{\min }\right)$. If $b_{c_{1}}=b_{\nabla}$ for all such $c_{1}>c$, we would have

$$
c \Vdash_{V^{(n)}} \chi^{b_{c}, \nabla}:=\bigwedge b_{c} \wedge\left(\bigvee\left(\operatorname{LitP}(r) \backslash b_{c}\right) \rightarrow \bigvee_{Y \in M} s_{k}(Y) \vee \phi(\nabla)\right),
$$

and $c \in e_{k+1}\left(X_{\nabla, b_{c}}\right)$ - a contradiction. Assume now that there are $c_{1}$ with $b_{c_{1}} \subset b_{\nabla}$, and choose a such $c_{1}$ with maximal $b_{c_{1}}$. So, $c_{1} \in\langle c\rangle \backslash e_{k}(\mathcal{M})$, and then if $c_{1} \leq c_{2}$ and $b_{c_{1}} \subset b_{c_{2}}$ then either $c_{2} \in e_{k}(\mathcal{M})$ or $b_{c_{2}}=b_{\nabla}$. Thus we obtain

$$
c_{1} \Vdash_{V^{(n)}} \chi^{b_{c_{1}}, \nabla}:=\bigwedge b_{c_{1}} \wedge\left(\bigvee\left(\operatorname{LitP}(r) \backslash b_{c_{1}}\right) \rightarrow \bigvee_{Y \in M} s_{k}(Y) \vee \phi(\nabla)\right),
$$

and $c_{1} \in e_{k+1}\left(X_{\nabla, b_{1}}\right)$, where $b_{c_{1}} \subset b_{\nabla}$. So, $c_{1} \in e_{k+1}\left(X\left(c_{1}, k+1\right)\right)$, where $X\left(c_{1}, k+1\right)=X_{\nabla, b_{c_{1}}}$ (cf. Proposition 5.4) and $X_{\nabla, b_{c_{1}}} \notin \nabla$ since $b_{c_{1}} \subset b_{\nabla}$. This contradicts $M^{k}(c)=M^{k+1}(c)=\nabla$. Thus (p3) for $k+1$ is verified.

For (p4):

We define the valuation $V_{k+1}$ as follows. For elements of $\operatorname{LitP}(r)$ it coincides with $V^{(n)}$ and for literals $\alpha$ of the form $z$ and $\sim z$, where $z$ is a variable of $r$, we set

$$
V_{k+1}(\alpha):=\bigcup_{0 \leq j \leq k+1}\left\{e_{j}(Y) \mid \alpha \in Y, Y \in M\right\} .
$$

From the definition of sets $e_{k+1}(Y), Y \in M$, it follows that the valuation $V_{k+1}$ is definable, extends $V_{k}$ and coincides with $V^{(n)}$ on $\operatorname{Lit} P(r)$. We need to show that $V_{k+1}$ coincides with $V_{l}$ for $l \leq k$ on $e_{l}(\mathcal{M})$.

Let $b \in e_{l}(Y)$ and $b \Vdash_{V_{l}} \alpha$, then $b \in e_{l-j}\left(Y_{1}\right)$ and $\alpha \in Y_{1}$. Then $b \in$ $e_{k+1}\left(Y_{1}\right)$ and $b \Vdash_{V_{k+1}} \alpha$. Conversely, let $b \in e_{l}(Y) \backslash e_{l-1}(\mathcal{M})$ and $b \Vdash_{V_{k+1}} \alpha$ (the case of other $l$ may evidently be reduced to this one by IH for (p4)). Than $b \in e_{k+1}\left(Y_{1}\right)$ for some $Y_{1}$, where $\alpha \in Y_{1}$. If $b \in e_{k}\left(Y_{1}\right)$ by IH for (p4) we have $b \Vdash_{V_{l}} \alpha$. If $b \in e_{k+1}\left(Y_{1}\right) \backslash e_{k}\left(Y_{1}\right)$ than $b \Vdash_{V^{(n)}} \psi^{\nabla}$ for some $\nabla$. Besides by $(\mathrm{p} 2) b \in e_{l}(X(b, l)) \backslash e_{l-1}(\mathcal{M})$ and hence $X(b, l) \in \nabla$. From $\alpha \in Y_{1}$ and $Y_{1}=X_{\nabla, b_{\nabla}}^{\min }$ or $Y_{1}=X_{\nabla, b}$ (cf. definition of $\left.e_{k+1}\left(Y_{1}\right)\right)$ we obtain $\alpha \in X(b, l)$. 
From $b \in e_{l}(X(b, l))$ and $\alpha \in X(b, l)$ we conclude $b \Vdash_{V_{l}} \alpha$. Thus (p4) is verified.

\section{For (p5):}

We need to show $\forall a \in e_{k+1}(\mathcal{M}) \backslash e_{k}(\mathcal{M})$,

$$
\forall \varphi \in S u b(r)\left[a \Vdash_{V_{k+1}} \varphi \Leftrightarrow[\varphi \in X(a, k+1)]\right] .
$$

If $a \in e_{k+1}(Y) \backslash e_{k}(\mathcal{M})$, by $(\mathrm{p} 2)$ for $(\mathrm{k}+1)$ and Proposition 5.4, we have $a \in e_{k+1}(X(a, k+1))$, where, for $\nabla=M^{k}(a)$, $e_{k}(\mathcal{M})$

(i) $X(a, k+1)=X_{\nabla, b_{\nabla}}^{\min }$ and $X_{\nabla, b_{\nabla}}^{\min } \supseteq Y$ for all $Y$, where $a \in e_{k+1}(Y) \backslash$

or (disjointly)

(ii) $Y=X(a, k+1)=X_{\nabla, b}$ for a unique $X_{\nabla, b}$, where $b \subseteq b_{\nabla}$.

Therefore by definition of $V_{k+1}(5.4)$ holds for every literal $\varphi \in S u b(r)$. We continue our proof by induction on the length of $\varphi$. Inductive steps for $\wedge$ and $\vee$ are evident, we do now $\rightarrow$.

\section{Direction: $\Leftarrow$}

Let $\beta_{1} \rightarrow \beta_{2} \in X(a, k+1)$. Let $a<c$ and $c \in e_{k}(\mathcal{M})$. Then $c \in$ $e_{j}(X(c, j)) \backslash e_{j-1}(\mathcal{M})$ for some $0 \leq j \leq k$ (recall that $\left.e_{-1}(\mathcal{M})=\varnothing\right)$ by $(\mathrm{p} 2)$ for $k$.

Therefore we obtain $X(c, j) \in \nabla$ and because $\beta_{1} \rightarrow \beta_{2} \in X(a, j+1)$ we also have $\beta_{1} \rightarrow \beta_{2} \in X(c, j)$ (cf. description of $X(a, k+1)$ above). Therefore by IH for (p5) we obtain: $c \Vdash_{V_{j}} \beta_{1} \rightarrow \beta_{2}$ and consequently $c \Vdash_{V_{k+1}} \beta_{1} \rightarrow \beta_{2}$.

Assume now that $a \leq c$ and $c \notin e_{k}(\mathcal{M})$. Then $c \in e_{k+1}(X(c, k+1)) \backslash$ $e_{k}(\mathcal{M})$ by Proposition 5.4.

If the case (i): $X(a, k+1)=X_{\nabla, b_{\nabla}}^{\min }$, then $a \Vdash_{V^{(n)}} \varphi_{1}\left(\nabla, b_{\nabla}\right)$. Since $a \leq c$, we have $c \Vdash_{V^{(n)}} \varphi_{1}\left(\nabla, b_{\nabla}\right)$ and $c \in e_{k+1}\left(X_{\nabla, b_{\nabla}}^{\min }\right) \backslash e_{k}(\mathcal{M})$ and by Proposition 5.4 for $c$ and properties of $X_{\nabla, b_{\nabla}}^{\min }$ we obtain $X(c, k+1)=X(a, k+1)$ and hence $\beta_{1} \rightarrow \beta_{2} \in X(c, k+1)$. Consequently, if $c \Vdash_{V_{k+1}} \beta_{1}$ then by $\mathrm{IH} \beta_{1} \in X(c, k+1)$ and hence by $\beta_{1} \rightarrow \beta_{2} \in X(c, k+1)$ we obtain $\beta_{2} \in X(c, k+1)$, which by IH implies $c \Vdash_{V_{k+1}} \beta_{2}$. Thus $a \Vdash_{V_{k+1}} \beta_{1} \rightarrow \beta_{2}$.

Assume now that we have case (ii): only the case $\mathrm{Y}=X(a, k+1)=X_{\nabla, b}$ is possible where $b \subseteq b_{\nabla}$ and $X_{\nabla, b}$ to be single, unique.

Then it could be two options for $c$ : (a1) $c \in e_{k+1}\left(X_{\nabla, b_{\nabla}}^{\min }\right) \backslash e_{k}(\mathcal{M})$ or (a2) $c \in e_{k+1}(X(a, k+1))$.

In case (a1) reasoning as above we conclude $c \Vdash_{V_{k+1}} \beta_{1} \rightarrow \beta_{2}$. In case (a2), if $c \leq c_{1} \notin e_{k}(\mathcal{M})$, then either $c_{1} \in e_{k+1}\left(X_{\nabla, b_{\nabla}}^{\min }\right) \backslash e_{k}(\mathcal{M})$, and reasoning as above we obtain $c_{1} \Vdash_{V_{k+1}} \beta_{1} \rightarrow \beta_{2}$. Or $c_{1} \in e_{k+1}(X(a, k+1))$, and applying IH we again conclude $c_{1} \Vdash_{V_{k+1}} \beta_{1} \rightarrow \beta_{2}$. In total, we proved, $a \Vdash_{V_{k+1}} \beta_{1} \rightarrow \beta_{2}$.

\section{Direction: $\Rightarrow$}

For opposite direction, assume now that $\beta_{1} \rightarrow \beta_{2} \notin X(a, k+1)$. Then by our choice of $X_{\nabla, b}$ and $X_{\nabla, b \nabla}^{\min }$ either (1) $\beta_{1} \in X(a, k+1)$ and $\beta_{2} \notin X(a, k+1)$, or (2) $\beta_{1} \rightarrow \beta_{2} \notin Y_{1}$ for some $Y_{1} \in \nabla=M^{k}(a)$. In the case (1) we have by induction hypothesis $a \Vdash_{V_{k+1}} \beta_{1}$ and $a \nVdash_{V_{k+1}} \beta_{2}$, so $a \nVdash_{V_{k+1}} \beta_{1} \rightarrow \beta_{2}$. 
Suppose we have the case (2) and $\beta_{1} \rightarrow \beta_{2} \notin Y_{1}$ for some $Y_{1} \in \nabla$. Since $\nabla=M^{k}(a)$, there are $c$ and $j \leq k$ such that $a \leq c, c \in e_{j}(X(c, j)) \backslash e_{j-1}(\mathcal{M})$ and $Y_{1}=X(c, j)$. By IH for $c$ (since $\left.j \leq k\right)$ we obtain $c \nVdash_{V_{j}} \beta_{1} \rightarrow \beta_{2}$. consequently $c \nVdash_{V_{k+1}} \beta_{1} \rightarrow \beta_{2}$ and $a \nVdash_{V_{k+1}} \beta_{1} \rightarrow \beta_{2}$

This completes proof of Lemma 5.3.

By (p3) our procedure terminates at a step $k$, where $k \leq m$ and $m$ is the number of worlds in $M$, and we get $e_{k}(\mathcal{M})=\mathcal{N}^{\mathbf{3}}(n)$. More exactly, we may stop at the first $k$ such that $\bigvee_{X \in M} s_{k}(X) \in \mathbf{N 3}$. Due to (p5) we have $V_{k}(\varphi(\bar{x}, \sim \bar{x}, \bar{p}, \sim \bar{p}))=N^{\mathbf{3}}(n)$ and $V_{k}(\psi(\bar{x}, \sim \bar{x}, \bar{p}, \sim \bar{p})) \neq N^{3}(n)$. We know that $V_{k}$ agrees with $V^{(n)}$ on $\bar{p}$ and $\sim \bar{p}$ and that the cones $V_{k}\left(x_{j}\right)$ and $V_{k}\left(\sim x_{j}\right), x_{j} \in \bar{x}$, are definable in $\mathcal{N}^{\mathbf{3}}(n)$ by formulas, say, $\bar{\xi}$ and $\bar{\zeta}$, i.e., $V_{k}\left(x_{j}\right)=V^{(n)}\left(\xi_{j}\right)$ and $V_{k}\left(\sim x_{j}\right)=V^{(n)}\left(\zeta_{j}\right), x_{j} \in \bar{x}$. In this way,

$$
V^{(n)}(\varphi(\bar{\xi}, \bar{\zeta}, \bar{p}, \sim \bar{p}))=V_{k}(\varphi(\bar{x}, \sim \bar{x}, \bar{p}, \sim \bar{p}))=N^{\mathbf{3}}(n)
$$

and

$$
V^{(n)}(\psi(\bar{\xi}, \bar{\zeta}, \bar{p}, \sim \bar{p}))=V_{k}(\psi(\bar{x}, \sim \bar{x}, \bar{p}, \sim \bar{p})) \neq N^{\mathbf{3}}(n)
$$

It remain to notice that $\neg(\bar{\xi} \wedge \bar{\zeta}) \in \mathbf{N 3}$. Assume that $V_{k}$ is not an N3valuation, i.e. that $x \Vdash_{V_{k}} p \wedge \sim p$ for some $x$ and $p$. Then $y \Vdash_{V_{k}} p \wedge \sim p$ for some maximal world $y$ over $x$. All maximal worlds of $\mathcal{N}^{\mathbf{3}}$ belong to $e_{0}(\mathcal{M})$, consequently by (p4) we have $y \Vdash_{V_{0}} p \wedge \sim p$. The latter is impossible, because we know that $V_{0}$ is an N3-valuation. We have thus proved that $V_{k}$ is an N3valuation. Consequently, $V^{(n)}\left(\xi_{j}\right) \cap V^{(n)}\left(\zeta_{j}\right)=\varnothing$, i.e. $V^{(n)}\left(\xi_{j} \wedge \zeta_{j}\right)=\varnothing$, whence $V^{(n)}\left(\neg\left(\xi_{j} \wedge \zeta_{j}\right)\right)=N^{\mathbf{3}}(n)$. The latter means that $\neg\left(\xi_{j} \wedge \zeta_{j}\right) \in \mathbf{N} \mathbf{3}$ for all $x_{j} \in \bar{x}$.

Assume now that we start from an $\mathbf{N} 4^{\mathrm{N}}$-model $\mathcal{M}$. We argue as above with the following two exceptions. First, we change the definition of the valuation $V$ on literal $\sim p_{X}$. For a maximal world $x$, the literal $\sim p_{X}$ is true at $x$ iff $p_{X}$ is false at $x$. If $x$ is not maximal, then $\sim p_{x}$ is false at $x$. Second, we have to notice that defining model $e_{0}(\mathcal{M})$ for every $c \in S_{1}\left(\mathcal{N}^{N}\right) \backslash e(\mathcal{M})$ we can choose a world $X_{0}(c, 0)$ maximal in $\mathcal{M}$ (see the proof of Proposition 5.1). All maximal worlds of $\mathcal{M}$ are complete, because this is an $\mathbf{N} 4^{\mathrm{N}}$-model. Consequently all maximal worlds of $e_{0}(\mathcal{M})$ and of every $e_{j}(\mathcal{M})$ are complete too. As a result we obtain an $\mathbf{N} 4^{\mathrm{N}}$-valuation $V_{1}$. This means that $V^{(n)}\left(\xi_{j} \vee \zeta_{j}\right)$ contains all maximal worlds of $N^{\mathrm{N}}(n)$, i.e. $\neg \neg\left(\xi_{j} \vee \zeta_{j}\right) \in \mathbf{N} 4^{\mathrm{N}}$.

Suppose that now it is clear how to treat an $\mathbf{N} 4^{\circ}$-model $\mathcal{M}$. AAAAAA

These remarks complete the proof of our Theorem 5.2.

From Proposition 5.1 and Theorem 5.2 we immediately obtain

Corollary 5.5 (Algorithmic criterion for weak admissibility in $\mathrm{N} 3\left(\mathrm{~N} 4^{\mathrm{N}}, \mathrm{N} 4^{\circ}\right)$ ). The rule with parameters $r=\varphi(\bar{x}, \sim \bar{x}, \bar{p}, \sim \bar{p}) / \psi(\bar{x}, \sim \bar{x}, \bar{p}, \sim \bar{p})$ is weakly admissible in $\mathbf{N} 3\left(\mathbf{N} 4^{\mathrm{N}}, \mathbf{N} 4^{\circ}\right)$ iff at least one of the conditions (a)-(c) from Proposition 5.1 is falsified in $\mathcal{M}=\langle M, \subseteq, Q, V\rangle$ for every $M \subseteq 2^{\text {Sub(r) }}$ such that $\mathcal{M}$ is an $\mathbf{N} 3-\left(\mathbf{N} 4^{\mathrm{N}}-, \mathbf{N}^{\circ}{ }^{-}\right)$model. 
Recall that a rule $r$ is said to be true on a frame w.r.t. a valuation $V$, if the conclusion of this rule is true w.r.t. $V$ at all worlds from this frame, when this holds for all formulas of the premise of $r$. From Proposition 5.1 and Theorem 5.2 we also immediately infer

Corollary 5.6 (Semantic criterion for weak admissibility in $\mathbf{N} 3\left(\mathbf{N} 4^{\mathrm{N}}, \mathbf{N} 4^{\circ}\right)$ ). The rule with parameters $r=\varphi(\bar{x}, \sim \bar{x}, \bar{p}, \sim \bar{p}) / \psi(\bar{x}, \sim \bar{x}, \bar{p}, \sim \bar{p})$ is weakly admissible in $\mathbf{N 3}\left(\mathbf{N} 4^{\mathrm{N}}, \mathbf{N} 4^{\circ}\right)$ iff $r$ is true on the frames of all models $\mathcal{N}^{\mathbf{3}}(n)$ $\left(\mathcal{N}^{\mathrm{N}}(n), \mathcal{N}^{\circ}(n)\right)$, with $P_{n} \supset P(r)$, w.r.t. all valuations coinciding with the original valuations of the models $\mathcal{N}^{3}(n)\left(\mathcal{N}^{\mathrm{N}}(n), \mathcal{N}^{\circ}(n)\right)$ at all literals from $\operatorname{Lit} P(r)$.

\section{References}

[1] A. Almukdad, D. Nelson, Constructible falsity and inexact predicates. J. Sym. Logic 49 (1984), 231-233.

Publishers, 2001, 447-533.

[2] S. Babenyshev, V. Rybakov, Linear Temporal Logic LTL: Basis for Admissible Rules. J. Log. Comput. 21(2) (2011), 157-177.

[3] S. Babenyshev, V. Rybakov, Unification in linear temporal logic LTL. Ann. Pure Appl. Logic 162(12) (2011), 991-1000.

[4] R. Iemhoff, On The Admissible Rules of Intuitionistic Propositional Logic. J. Symb. Log. 66(1) (2001), 281-294.

[5] R. Iemhoff, Towards a Proof System for Admissibility. CSL-2003 (2003), 255270.

[6] R. Iemhoff, G. Metcalfe, Proof theory for admissible rules. Ann. Pure Appl. Logic 159(1-2) (2009), 171-186.

[7] E. Jerabek, Bases of admissible rules of Lukasiewicz logic. J. Log. Comput. 20(6) (2010), 1149-1163.

[8] E. Jerabek, Admissible rules of Lukasiewicz logic. J. Log. Comput. 20(2) (2010), 425-447.

[9] E. Jerabek, Independent bases of admissible rules. Logic Journal of the IGPL 16(3) (2008), 249-267.

[10] E. Jerabek, Complexity of admissible rules. Arch. Math. Logic 46(2) (2007), 73-92.

[11] E. Jerabek, Admissible rules of modal logics. J. Log. Comput. 15(4) (2005), 411-431.

[12] S. Ghilardi, Unification in intuitionistic logic. J. Symb. Log. 64(2) (1999), 859880.

[13] S. Ghilardi, Best solving modal equations. Ann. Pure Appl. Logic 102(3) (2000), 183-198.

[14] Y. Gurevich, Intuitionistic logic with strong negation. Stud. Log. 36(1-2) (1977), 49-59.

[15] H. Friedman, One Hundred and Two Problems in Mathematical Logic. J. Symb. Logic 40(3) (1975), 113-130. 
[16] I. Johansson, Der Minimalkalkül, ein reduzierter intuitionistischer Formalismus. Compositio Mathematika 4 (1937), 119-136.

[17] E.I.Latkin, Generalized Kripke Semantics for Nelson's Logic. Algebra and Logic 49(5) (2010), 426-443.

[18] E.G.K. López-Escobar, Refutability and elementary number theory. Indag. Math. 34 (1972), 362-374.

[19] D. Nelson, Constructible falsity. J. Symb. Logic 14(2) (1949),16-26.

[20] S.P. Odintsov, Algebraic semantics for paraconsistent Nelson's logic. J. Log. Comput. 13(4) (2003), 453-468.

[21] S.P. Odintsov, The Class of Extensions of Nelson's paraconsistent logic. Stud. Log. 80(2-3) (2005), 293-322.

[22] S.P. Odintsov, Constructive negations and paraconsistency. Springer, Dordrecht, 2008.

[23] S. Odintsov, D. Pearce, Routley semantics for answer sets. In Proc. 8th International Conference on Logic Programming and Nonmonotonic Reasoning, LNCS 3662, 2005, 343-355.

[24] S.P. Odintsov, V.V. Rybakov, Unification and admissible rules for paraconsistent minimal Johannson's logic $\mathbf{J}$ and positive intuitionistic logic $\mathbf{I P C}^{+}$. Ann. Pure Appl. Logic 164 (2013), 771-784.

[25] G. Priest, Paraconsistent logic. Stanford Encyclopedia of Philosophy (http://plato.stanford.edu/entries/logic-paraconsistent/).

[26] H. Rasiowa, $N$-lattices and constructive logic with strong negation. Fund. Math. 46 (1958), 61-80.

[27] H. Rasiowa, An algebraic approach to non-classical logic. PWN, Warsaw and North-Holland, Amsterdam, 1974.

[28] R. Routley, Semantical analyses of propositional systems of Fitch and Nelson. Stud. Log. 33(3) (1974), 283-298.

[29] V. Rybakov, A criterion for admissibility of rules in the modal system $\mathrm{S}_{4}$ and the intuitionistic logic. Algebra and Logica 23(5) (1984), 369-384.

[30] V.V. Rybakov, Problems of Substitution and Admissibility in the Modal System Grz and in Intuitionistic Propositional Calculus. Ann. Pure Appl. Logic 50(1) (1990), 71-106.

[31] V.V. Rybakov, Rules of inference with parameters for intuitionistic logic. J. Symb. Logic 57(3) (1992), 912-923.

[32] V. V. Rybakov, Construction of an Explicit Basis for Rules Admissible in Modal System S4. Math. Log. Q. 47(4) (2001), 441-446.

[33] V.V. Rybakov, Logics with the universal modality and admissible consecutions. J. Appl. Non-Class. Logics 17(3) (2007), 383-396.

[34] V.V. Rybakov, Linear temporal logic with until and next. Logical consecutions. Ann. Pure Appl. Logic 155(1) (2008), 32-45.

[35] V.V. Rybakov, Multi-modal and Temporal Logics with Universal Formula Reduction of Admissibility to Validity and Unification. J. Log. Comput. 18(4) (2008), 509-519.

[36] V.V. Rybakov, Rules admissible in transitive temporal logic TS4, sufficient condition. Theor. Comput. Sci. 411(50) (2010), 4323-4332. 
[37] R.H. Thomason, A semantical study of constructible falsity. Z. Math. Logik Grundl. Math. 15 (1969), 247-257.

[38] N.N. Vorob'ev, A constructive propositional calculus with strong negation. Doklady Akademii Nauk SSSR 85 (1952), 465-468.

[39] N. N. Vorob'ev, The problem of deducibility in constructive propositional calculus with strong negation. Doklady Akademii Nauk SSSR 85 (1952), 689-692.

[40] H.Wansing, Negation. in L. Goble (ed.), The Blackwell Guide to Philosophical Logic, Cambridge, MA: Basil Blackwell Publishers, 2001, 415-436.

\author{
Sergei Odintsov \\ Institute of Mathematics, \\ 4 Acad. Koptyug avenue, \\ 630090, Novosibirsk, \\ Russian Federation \\ e-mail: odintsov@math.nsc.ru \\ Vladimir Rybakov \\ School of Computing, Mathematics and DT, \\ Manchester Metropolitan University, \\ John Dalton Building, Chester Street, \\ Manchester M1 5GD, U.K. \\ e-mail: V.Rybakov@mmu.ac.uk
}

